\title{
Ankyrin G Organizes Membrane Components to Promote Coupling of Cell Mechanics and Glucose Uptake
}

\author{
Alicia M. Salvi, Jennifer L. Bays, Samantha R. Mackin, René-Marc Mege ${ }^{\#}$, Kris A. DeMali \\ Department of Biochemistry, University of lowa Roy J. and Lucille A. Carver College of Medicine, \\ lowa City, IA 52242.
}

\begin{abstract}
The response of cells to forces is critical for their function and occurs via rearrangement of the actin cytoskeleton ${ }^{1}$. Cytoskeletal remodeling is energetically costly ${ }^{2,3}$, yet how cells signal for nutrient uptake remains undefined. In this study, we present evidence that force signals for increased glucose uptake by stimulating glucose transporter 1 (GLUT1). GLUT1 recruitment to and retention at sites of force transmission requires non-muscle myosin IIA- mediated contractility and ankyrin G. Ankyrin G forms a bridge between the force transducing receptors and GLUT1. This bridge is critical for allowing cells under tension to tune glucose uptake to support remodeling of the actin cytoskeleton and forming an epithelial barrier. Collectively, these data reveal an unexpected mechanism for how cells under tension take up nutrients and provide insight into how defects in glucose transport and mechanics might be linked.
\end{abstract}

\section{Results:}

To begin to identify how cells take up glucose in response to force, cells were treated with pharmacological inhibitors targeting two glucose transporters (GLUTs) present in epithelial cells, GLUT1 and GLUT4. For this, shear stress was applied to monolayers of MCF-10A or MDCKII epithelial cells ${ }^{4,5}$. In response to shear stress, a significant increase in glucose uptake was observed in both MDCKII (Figure 1a) and MCF-10A (Figure 1b) cells. Preincubation of the cells with WZB117, an inhibitor of GLUT1, prevented the glucose uptake (Figure 1a and 1b). This inhibition was specific to GLUT1 as a GLUT4 inhibitor, indinivar, had no effect on glucose uptake (Figure 1a and b). Lastly, the force-stimulated glucose uptake was blocked by blebbistatin, a myosin II inhibitor, suggesting a requirement for contractility (Figure 1a and b).

To more rigorously test the requirement for GLUT1, GLUT1 expression was stably inhibited using two different shRNAs in the MDCKII and MCF-10A cells. A scramble sequence

\footnotetext{
"Corresponding author: Kris DeMali, Department of Biochemistry, University of Iowa Carver College of Medicine, Iowa City, IA 52242. kris-demali@uiowa.edu; tel:319-335-7882.

\#Université de Paris, CNRS, Institut Jacques Monod, 75013 Paris, France.

Contributions: A.S designed and performed experiments, analyzed data, and wrote the manuscript. JLB and SRM performed experiments. KAD aided the experimental design, wrote the manuscript, and directed the project. RMM provided reagents. All authors provided detailed comments.

Competing Interest

The authors declare no competing interests
} 
(scrGLUT1) was included as a control. Using this approach, GLUT1 expression was inhibited by $43 \%$ with one of the shRNAs (shGLUT1.1) and 53\% by the second shRNA (shGLUT1.2) in MDCKII cells (Figure 1c). A 40\% and 56\% inhibition of GLUT1 were achieved in MCF-10A cells expressing shGLUT1.1 and shGLUT1.2, respectively (Figure 1d). An examination of glucose uptake in these cells revealed that shear stimulated glucose uptake in the parental and scramble-expressing MDCKII (Figure 1e) and MCF-10A cells (Figure 1f). This effect was abrogated in the MDCKII and MCF-10A cells expressing the shRNAs against GLUT1 (Figure 1e and f). Stable inhibition of another glucose transporter, GLUT3, had no effect on force stimulated glucose uptake (Extended Data Figure 1a and b).

To ensure the increased glucose uptake was not dependent on shear, an alternative form of force was employed. For this, cells were incubated with magnetic beads coated with E-cadherin extracellular domains (or IgG as a control), and tensile forces were applied as previously described ${ }^{6-13}$. Application of tensile forces stimulated an increase in glucose uptake in the MDCKII (Figure 1g) and MCF-10A cells (Figure 1h and Extended Data Figure 1c). Importantly, glucose uptake was GLUT1-dependent (Figure 1g-h and Extended Data Figure 1c). GLUT4 was not involved (Figure 1g and Extended Data Figure 1c).

To ensure that the glucose uptake stimulated by force was not a consequence of culturing cells on stiff substrates, glucose uptake in cells plated on soft elastic collagen matrices ( storage modulus $\left(\mathrm{G}^{\prime}\right)=16.1 \mathrm{~Pa}$, loss modulus $\left(\mathrm{G}^{\prime \prime}\right)=2.7 \mathrm{~Pa}$ ) was examined ${ }^{14}$. A similar magnitude of glucose uptake was observed on soft substrates (Extended Data Figure 1d) and in cells plated on stiff tissue culture substrates (Figure 1a). Taken together, these data demonstrate that GLUT1 specifically increases glucose uptake in cells exposed to force.

To define how GLUT1 changes in response to force, its localization in cells exposed to force was examined. For this, MDCKII cells were subjected to shear, and GLUT1 localization was examined. In the absence of shear stress, there was some GLUT1 in the cytoplasm and some co-localization was observed between GLUT1 and $\beta$-catenin, a marker of cell-cell junctions (Figure 2a). Application of shear stress stimulated a $2.5 \pm 0.2$-fold enrichment of $\beta$-catenin (Figure 2b) and a 5.5 \pm 2.0 fold increase in GLUT1 (Figure 2c) in the cell-cell junctions in parental cells (Figure 2a-c). The scramble expressing cells showed a similar effect (Extended Data Figure 1e-g). This observation agrees with published findings showing junctional GLUT1 localization in tissues ${ }^{15-17}$. Orthogonal sections revealed co-localization of GLUT1 with $\beta$-catenin in cell-cell junctions was increased when cells were exposed to force (Extended Data Figure 1h). In contrast, in cells with depressed levels of GLUT1, little GLUT1 was observed in cell-cell junctions (Figure 2a and c). To examine whether surface expression of GLUT1 was altered by shear, surface proteins were biotinylated and examined by immunoblotting. In the absence of shear, little GLUT1 appeared in the biotinlabeled fraction. Shear induced a small, but significant 1.9 \pm 0.3 -fold increase in surface levels of GLUT1 (Figure 2d). The enrichment of GLUT1 in cell-cell junctions was not the consequence of an increase in GLUT1 expression (Extended Data Figure 1i). Taken together with the immunofluorescence data, these experiments reveal that there is a small increase in total surface levels of GLUT1 in response to shear, but the amount of GLUT1 in the cell-cell junctions increases dramatically. 
To determine if E-cadherin was required for GLUT1 enrichment in cell-cell junctions, we examined GLUT1 localization in published cell lines lacking E-cadherin (shEcad) ${ }^{18}$. Consistent with published findings, cells formed $\beta$-catenin containing cell-cell junctions in the absence of shear stress (Figure $2 a-b)^{18}$. These junctions also contained GLUT1 (Figure 2a), and the amount of GLUT1 in cell-cell junctions did not statistically change when force was applied (Figure 2a and 2c). Thus, E-cadherin is required for force stimulated enrichment of GLUT1 in cell-cell junctions.

To test if GLUT1 co-localization to cell-cell junctions was limited to cells in 2D cultures, MDCKII cells were embedded in a 3D extracellular matrix of Matrigel. Consistent with published reports, MDCKII cells formed a spherical cyst with a hollow lumen surrounded by a monolayer of polarized cells that resemble that of epithelia in vivo (Figure 2e) ${ }^{19-21}$. Under these conditions, strong GLUT1 co-localization with E-cadherin was observed in the cells on the cyst exterior and with the basolateral surface of the cells on the cyst interior (Figure 2e). Interestingly, strong GLUT1 staining was also observed on the apical surface of the cyst lumen. Thus, GLUT1 co-localization with E-cadherin occurs in 3D (Figure 2e).

The requirement for E-cadherin for GLUT1 enrichment in cell-cell junctions (Figure 2a) suggested that GLUT1 might be integrating into the cadherin adhesion complex. To test this possibility directly, E-cadherin was immunoprecipitated in the presence or absence of shear, and GLUT1 association was examined by immunoblotting. In the absence of shear, some GLUT1 co-immunoprecipitated with E-cadherin in the control cells (Figure 2f). The amount of GLUT1 that co-immunoprecipitated increased significantly upon exposure to shear. In contrast, in the cells with depressed levels of GLUT1, there was little GLUT1 bound to Ecadherin and the amount did not change when shear was applied (Figure 2f). To determine if force transmission was required, the cells were pre-incubated with blebbistatin. Under these conditions, GLUT1 co-immunoprecipitation with E-cadherin was blocked (Figure 2f). Taken together, these data suggest that GLUT1 is recruited to and integrates in cadherin-containing cell-cell junctions in response to shear.

Glucose enters cells in response to force and is metabolized into ATP, which in turn provides the energy necessary to support the growth of the cadherin adhesion complex and reinforcement of the actin cytoskeleton in order for the cell to resist force ${ }^{11}$. This process is known as cell stiffening. To begin to determine the role of GLUT1 in this process, the time course of reinforcement was examined. For this, MDCKII cells were subjected to shear stress for 2 hours, and the cells were stained with antibodies against E-cadherin or phalloidin. Both E-cadherin (Extended Data Figure 2a-b) and F-actin (Extended Data Figure $2 \mathrm{a}$ and $\mathrm{c}-\mathrm{f}$ ) were enriched in cell-cell junctions immediately after application of shear (0 minutes) and 30 minutes of recovery from shear, suggesting that reinforcement was occurring. F-actin enrichment in cell-cell junctions was significantly decreased 1 hour after shear (Extended Data Figure 2a and 2c). In contrast, E-cadherin enrichment persisted for 4 hours (Extended Data Figure 2a and b). By 6 hours of recovery, both E-cadherin and F-actin had returned to prestress levels (Extended Data Figure 2a-c). Based on these observations, actin and E-cadherin were examined immediately after application of shear in all subsequent studies. 
To investigate whether GLUT1 is required for cell stiffening, the effect that loss of GLUT1 has E-cadherin and actin reinforcement was examined. Shear stress increased both E-cadherin (Figure 3 a, Extended Data Figure 3a-b) and F-actin (Figure 3a-b, Extended Data Figure 3a) enrichment in cell-cell junctions by 3-fold in parental cells or in cells expressing a scrambled shRNA (Extended Data $2 \mathrm{~d}-\mathrm{f})^{11}$. The magnitude of the reinforcement was independent of plating the cells on stiff (Figure 3a) or soft matrices (Extended Data Figure 3c-e). Furthermore, inhibition of GLUT1 prevented E-cadherin and F-actin enriched in cell-cell junctions (Figure 3a-b, Extended Data 3a-b).

To ensure the that inhibition of GLUT1 was specific for the actin cytoskeletal rearrangements and not simply altering ATP dependent processes, the activity of two protein kinases involved in cell stiffening were examined ${ }^{11}{ }^{12}$. Both AMPK (Extended Data Figure 3f) and Abelson tyrosine kinase (Abl) (Extended Data Figure 3g) were unaltered by GLUT1 inhibition.

Finally, to determine if reinforcement required glucose to be metabolized, cells were preincubated with 3PO, a phosphofructokinase-2 inhibitor. 3PO blocked force-stimulated enrichment of F-actin (Figure 3c-d) and E-cadherin (Figure 3c, Extended Data Figure 3h). This observation is further supported by our published data demonstrating that incubation of cells in low glucose containing media or inhibition of ATP synthesis prevents cell stiffening in response to shear stress ${ }^{11}$. Taken together, these data demonstrate that GLUT1 recruitment to cell-cell junctions allows for glucose uptake—an event that is essential for reinforcement of the cytoskeleton.

The images in Figure 3 a suggested that the actomyosin structure induced by force is very similar in appearance to the junctional localized non-muscle myosin IIA stress fibers previously described by the Mege group ${ }^{22}$. To determine if non-muscle myosin IIA is required for reinforcement of the actin cytoskeleton in response to force, the cells employed in the Mege studies ${ }^{22}$ were obtained, and their ability to reinforce the actin cytoskeleton in response to shear was examined. These MDCK cells express GFP-E-cadherin with nonmuscle myosin IIA (shNMIIA) or IIB (shNMIIB) inhibited. A control cell line (shCtrl) was also examined. When shear was applied to the control cells or cells lacking nonmuscle myosin IIB, E-cadherin (Figure 3e, Extended Data figure 3i) and F-actin (Figure $3 \mathrm{e}-\mathrm{f}$ ) enrichment in cell-cell junctions were increased. In contrast, in the cells with nonmuscle myosin IIA inhibited, neither E-cadherin nor F-actin was enriched (Figure 3e-f and Extended Data Figure 3i). Thus, reinforcement requires non-muscle myosin IIA.

Given the roles of GLUT1 (Figure 3a) and non-muscle myosin IIA (Figure 3e-f) in reinforcement of the actin cytoskeleton, we examined their interdependency. For this, forcestimulated glucose uptake (Figure 3g) and GLUT1 localization to cell-cell junctions (Figures $3 \mathrm{~h}$ ) were monitored in the cells lacking non-muscle myosin II isoforms. The control and shNMIIB cell lines took up glucose in response to shear (Figure 3g). In contrast, glucose uptake was not increased in shNMIIA cells (Figure $3 \mathrm{~g}$ ). Similarly, in the absence of shear, there was little GLUT1 co-localization with E-cadherin (Figure 3h-j). Upon exposure to shear, robust enrichment of GLUT1 (Figure 3h-i) and E-cadherin (Figure 3h, j) were observed in the control cells and the cells lacking non-muscle myosin IIB. There was not 
a significant increase in enrichment of E-cadherin or GLUT1 in the cells with suppressed levels of non-muscle myosin IIA (Figure 3h-j). Thus, GLUT1 recruitment, glucose uptake, and reinforcement of the actin cytoskeleton are non-muscle myosin IIA-dependent.

Figure 2 indicates that GLUT1 is retained in cell-cell junctions in response to shear. How might GLUT1 be retained at the plasma membrane in response to tension? Ankyrin G emerged as a good candidate ${ }^{23,24}$ because it binds E-cadherin ${ }^{25-27}$, and it and its family members mediate the retention of many surface proteins at the plasma membrane via its linkages to the spectrin-actin cytoskeleton ${ }^{25}, 28-34$. To test whether ankyrin $\mathrm{G}$ was required for GLUT1 retention at cell-cell contacts, its sensitivity to force was examined. For this, epithelial cells were exposed to shear and localization of ankyrin $G$ to cell-cell junctions was examined. In the absence of shear, low levels of ankyrin $\mathrm{G}$ were observed (Figure 4a and $4 b)^{26,27}$ Upon application of shear, there was a $16 \pm 0.2$ fold enrichment of $\beta$-catenin and 2.8 \pm 0.4 -fold enrichment in ankyrin staining in cell-cell junctions (Figure $4 \mathrm{a}$ and $4 \mathrm{~b}$. This enrichment required E-cadherin expression (Figure $4 a-b$ ). In further support of a sensitivity to force, co-immunoprecipitation of ankyrin $\mathrm{G}$ with E-cadherin increased in response to shear (Figure 4c). Thus, ankyrin G recruitment to E-cadherin containing cell-cell junctions requires force.

To test if ankyrin G was required for GLUT1 recruitment to cell-cell contacts, cells with ankyrin G silenced (shANKG) or expressing a scrambled sequence (scrANKG) were generated. Using this approach, a 50\% reduction in ankyrin $\mathrm{G}$ levels was achieved (Figure 4d). Co-localization of GLUT1 with $\beta$-catenin in cell-cell junctions was examined using immunofluorescence. In these studies, GLUT1 was observed in cell-cell junctions in the absence of shear (Figure 4e and $\mathrm{f}$ ). Application of shear induced a robust recruitment of GLUT1 to cell-cell contacts, and this effect was completely blocked in cells lacking ankyrin G (Figure $4 e$ and f).

To determine if ankyrin G was required for GLUT1 functions identified in Figures 1 and 2, we examined if glucose uptake and cell stiffening required ankyrin G. Control scrANKG-expressing cells exhibited an increase in glucose uptake (Figure 4g), whereas shear stimulated glucose uptake was blocked in the shANKG expressing cells (Figure $4 \mathrm{~g})$. Additionally, the control cells expressing the scramble sequence reinforced their actin cytoskeletons and cell-cell junctions in response to shear, whereas the cells lacking ankyrin $\mathrm{G}$ did not (Extended Data Figure $4 \mathrm{a}-\mathrm{c}$ ). These observations suggest that ankyrin $\mathrm{G}$ is sensitive to force and is required for cells to recruit GLUT1 to cell-cell junctions and shear stress stimulated glucose uptake.

To understand how ankyrin G recruits GLUT1 to cell-cell junctions, co-immunoprecipitation of GLUT1 and ankyrin G with E-cadherin was examined. In control cells expressing a scramble sequence (scrANKG), both ankyrin G and GLUT1 co-precipitated with E-cadherin. Upon exposure to shear stress, the amount of ankyrin G and GLUT1 coprecipitating with E-cadherin increased significantly (Figure $4 \mathrm{~h}$ ). This finding is not simply the result of an increase in ankyrin $\mathrm{G}$ expression as the amount of ankyrin $\mathrm{G}$ does not change in either the soluble or insoluble fraction in response to shear (Extended Data Figure 4d). Strikingly, inhibition of ankyrin G completely blocked the force stimulated 
co-precipitation of GLUT1 with E-cadherin (Figure 4h). This data suggests a force-induced complex of E-cadherin, ankyrin G, and GLUT1 forms. How ankyrin G binds GLUT1 is not completely understood. Both proteins bind to the spectrin cytoskeleton ${ }^{31,35,36}$ and spectrin was enriched in cell-cell junctions in response to force (Extended Data Figure 4e). Taken together these data suggest that ankyrin G tethers GLUT1 to the cadherin adhesion complex.

Global inhibition of ankyrin $\mathrm{G}$ could have many effects ${ }^{25,26}$. To specifically probe its function, mutant $\mathrm{E}$-cadherins unable to bind ankyrin $\mathrm{G}$ were generated. For this, we employed a previously described E-cadherin PolyA mutant protein in which seven residues between amino acids 738-758 of human E-cadherin juxtamembrane region were mutated to alanine residues to prevent ankyrin $\mathrm{G}$ binding ${ }^{26}$. The PolyA E-cadherin mutant or a wildtype (WT) control E-cadherin protein were expressed to similar extents in cells lacking Ecadherin (Extended Data Figure 5a). The PolyA mutation prevented co-immunoprecipitation of E-cadherin and ankyrin G (Extended Data Figure 5b and c). Thus, the PolyA mutant is behaving as previously reported ${ }^{26}$

To identify the effects that this mutant has on GLUT1, the E-cadherin proteins were immunoprecipitated and co-precipitation of GLUT1 was monitored using immunoblotting. In contrast to the wildtype protein, which bound GLUT1 when shear was applied, the PolyA mutant did not bind GLUT1 (Figure 5a). An examination of these cells using immunofluorescence revealed similar findings (Figure 5b). Both the wildtype and PolyA mutant localized to cell-cell junctions to a similar extent in the absence of shear. When shear was applied, the levels of GFP-E-cadherin were enriched while the PolyA mutant remained at pre-shear levels. GLUT1 was highly enriched and co-localized with wildtype, GFP-E-cadherin in cell-cell junctions in response to shear, whereas GLUT1 did not localize to cell-cell junctions in cells expressing the PolyA mutant (Figure 5b-c). Taken together, these data suggest that ankyrin G binding to E-cadherin is required for GLUT1 enrichment at cell-cell junctions in response to force.

Estimates suggest that $50 \%$ of ATP within a cell is used to support the actin cytoskeleton 2,3 . During highly dynamic actin cytoskeletal events, cells increase their energy production ${ }^{37-39}$. To determine if ankyrin G is also required for GLUT1 function, the ability of the PolyA mutant to support force stimulated glucose uptake and ATP production were examined. Cells expressing wildtype E-cadherin protein showed a 2.8-fold increase in glucose uptake and a 1.8-fold increase in the amount of relative intracellular ATP when exposed to shear stress (Figure 5d and e). In contrast, cells expressing the PolyA mutant E-cadherin were defective in taking up glucose and elevating intracellular ATP levels in response to shear (Figure $5 \mathrm{~d}$ and e). Similarly, to determine if ankyrin $\mathrm{G}$ binding to E-cadherin is required for reinforcement of the actin cytoskeleton in response to application of shear stress, F-actin enrichment was monitored using immunofluorescence. Cells expressing the wildtype E-cadherin exhibit robust enrichment of actin cytoskeleton in response to shear (Figure 5f-h). In contrast, cells expressing the PolyA mutant E-cadherin had the same amount of F-actin in their cell-cell junctions in the presence and absence of force. Similarly, spectrin was not reinforced in response to shear in cells expressing the PolyA mutant (Extended Data $5 \mathrm{~d}-\mathrm{e})$. Taken together, these data indicate a requirement for ankyrin $\mathrm{G}$ binding to E-cadherin for reinforcement of the actin and spectrin cytoskeleton (Figure 5f-h). 
To assess the physiological significance of ankyrin G recruitment of GLUT1, the formation of an epithelial barrier was monitored in MDCKII cells after a calcium switch-this is an event highly dependent upon increased contractility ${ }^{11,40-42}$. After re-addition of calcium, cells expressing the wildtype E-cadherin gradually formed an epithelial barrier over the first ten hours (Figure 5i) ${ }^{11,40,42}$. By twenty-four hours, the barrier had remodeled, and a strong barrier with a resistance value similar to what has been reported was achieved ${ }^{11,40,42}$. In contrast, both the initial formation and the final resistance of the epithelial barrier were significantly impaired in the cells expressing the PolyA E-cadherin (Figure 5i). Taken together, these data indicate GLUT1 recruitment to cell-cell junctions is required for efficient formation of an epithelial barrier.

The data presented herein establishes a mechanism for how force stimulates the uptake of metabolites from the environment in order to fuel the cell's efforts to resist the strain. Our data identifies GLUT1 as critical for the uptake of glucose in response to force and demonstrates a requirement for ankyrin G to retain GLUT1 at sites of E-cadherin force transmission. It is important to note that since the PolyA mutant did not localize to cell-cell junctions in response to shear as well as the wildtype protein, there is the possibility that another E-cadherin function is required. Nonetheless, as a result, glucose can be taken up and converted to ATP that is used to fuel non-muscle myosin IIA-dependent reinforcement of the actin cytoskeleton. This information provides a key missing link in understanding how mechanotransduction derives fuel sources. Importantly, this new insight could explain the defects that might arise in disease and highlight improvements that can be made to prevent metabolic disturbances.

\section{Material/Methods:}

\section{Cells Lines}

MCF-10A human breast epithelial cells and MDCKII canine kidney epithelial cells were purchased from ATCC and were maintained as previously described ${ }^{11,12,41}$. All cell lines and their derivatives were used for no more than 12 passages and periodically checked for mycoplasma contamination. shGLUT1-1, shGLUT1-2, scrGLUT1, shGLUT31, shGLUT3-2, scrGLUT3 and scrANKG expressing cells were selected and maintained in puromycin $(2 \mu \mathrm{g} / \mathrm{mL})$. shANKG cells were selected and cultured in $\mathrm{G} 418$. shEcad/WT and shEcad/PolyA were selected and maintained in blasticidin $(3 \mu \mathrm{g} / \mathrm{mL})^{26}$. shCtrl, shNMIIA and shNMIIB expressing cells were maintained in media containing geneticin $(5 \mu \mathrm{g} / \mathrm{mL})$ and puromycin $(2.5 \mu \mathrm{g} / \mathrm{mL})$. The 293GPG and 293FT (Invitrogen) cells are virus-producing cell lines that are a derivative of $293 \mathrm{~T}$ cells. The 293 GPG were maintained as described previously ${ }^{8}$ and the 293FT were maintained in DMEM supplemented with $10 \%$ heatinactivated FBS, 0.1mM MEM Non-essential Amino Acids (NEAA), 6mM L-glutamine, 1mM MEM Sodium Pyruvate, $1 \%$ Pen-Strep. MDCKII cell lines expressing shEcad were previously described $^{18}$.

\section{Constructs}

To inhibit expression of proteins using RNA interference, two distinct sequences targeting the exon portion of each gene of interest were individually inserted into pSUPER-retro-puro 
(Oligoengine), the sequences targeted were, shGLUT1.1: 5'-

GATCCCCCACTGGAGTCATCAATGCCTTCAAGAGAGGCATTGATGACTCCAGTGT

TTTTA-3'; shGLUT1.2: 5'-

GATCCCCTGCTGATGATGAACCTGCTTTCAAGAGAAGCAGGUUCAUCAUCAGCAT

TTTTA-3'; scrGLUT1: 5'-

GATCCCCTTCTCCGAACGTGTCACGTTTCAAGAGAACGTGACACGTTCGGAGAAT

TTTTA-3'; shGLUT3.1: 5'

-GATCCCCCTTGGTCTTTGTAGCCTTCTTTTCAAGAGAAAGAAGGCTACAAAGACC

AAGTTTTTA-3; shGLUT3.2: 5'-

GATCCCCAGTAGCTAAGTCGGTTGAAATTTCAAGAGAATTTCAACCGACTTAGCTA

CTTTTTTA-3 ' ; scrGLUT3.: 5'-

GATCCCCGGCTTATTGTTCGCTTCTTCTTTCAAGAGAAGAAGAAGCGAACAATAA

GCCTTTTTA-3'; scrANKG: 5'-

GATCCCCAGCGTCGACGTTATCATAATCTTCAAGAGAGATTATGATAACGTCGACG

CTTTTTTC-3'. cDNAs containing GFP-tagged wild-type E-cadherin or Poly A E-cadherin containing 7 amino acid changes in the ankyrin G binding site were previously described and the generous gift of Dr. Paul Jenkins ${ }^{26}$. Specifically, the amino acid sequence corresponding with human CDH1 (738-758) was mutated from:

KEPLLPPEDDTRDNVYYYDEE to AEPLLPPAAATRANVYYADEA.

\section{Virus Production and infection}

Retroviruses were produced as previously described ${ }^{12}$. To produce lentivirus, 293FT cells were transfected and viruses were produced according to the manufacturer's instructions (Invitrogen, R70007). Prior to infection, MCF-10A or MDCKII cells were grown to 75\% confluency. On day of infection, cells were washed 2x with serum-free DMEM, incubated in OPTIMEM (GibcoBRL) plus $4 \mu \mathrm{g} / \mathrm{mL}$ of polybrene, and concentrated viral particles were added to the cultures for $4 \mathrm{hrs}$ at $37^{\circ} \mathrm{C}$. Growth media was then added to the cultures, and $12-16 \mathrm{~h}$ later, the cells were placed in growth media plus drug.

\section{Magnetic Bead Assay}

The application of tensile force to E-cadherin using magnetic beads was performed as previously described ${ }^{8}$. Briefly, for E-cadherin and IgG beads, $1.5 \mu \mathrm{g}$ of paramagnetic Dynabeads Protein A (Invitrogen) were coated with $10 \mu \mathrm{g}$ of E-cadherin or IgG for 12 hours at $4{ }^{\circ} \mathrm{C}^{7}, 11$. The beads were washed in phosphate buffered saline (PBS) and blocked in 5\% bovine serum albumin (Sigma) in PBS. Coated beads were incubated with $90 \%$ confluent cells for 1 hour at $37^{\circ} \mathrm{C}$. Tensile force was applied for 10 minutes, by placing the cells 0.6 $\mathrm{cm}$ from a permanent ceramic magnet at room temperature. The cells were transferred to ice, washed, and lysed.

\section{Orbital Shear Stress}

Dishes of 90-95\% confluent cells with growth media were rotated on a New Brunswick orbital shaker at $200 \mathrm{rpm}$ for $2 \mathrm{~h}$ at $37^{\circ} \mathrm{C}$. The cells were transferred to ice and immediately lysed or fixed in paraformaldehyde. 


\section{Inhibitors and Functioning Blocking Antibodies}

Inhibitors were incubated with the cells immediately before shear and during the experiment. For tensile force studies, inhibitors were pre-incubated with cells for $2 \mathrm{~h}$ prior to application of tensile force. GLUT1 was inhibited using 30 WZB117 (Tocris Biosciences). Myosin II was inhibited using 50 $\mu \mathrm{M}$ blebbistatin (Sigma). E-cadherin was inhibited using a function blocking antibody, HECD-1 (Invitrogen) at $200 \mu \mathrm{g} / \mathrm{mL}$, and PFK2 was inhibited by at $50 \mu \mathrm{M}$ of 3PO (Millipore).

\section{Surface Biotinylation}

Surface proteins were labelled with EZ-Link ${ }^{\mathrm{TM}}$ NHS-LC-Biotin (Thermo Scientific; 21335) as previously described ${ }^{43}$. Dynabeads ${ }^{\mathrm{TM}} \mathrm{M}-280$ Streptavidin (Invitrogen; 11205D) were used to recover biotinylated proteins. Surface and total cellular levels of GLUT1 were examined by immunoblotting.

\section{D Culture of Cysts}

Matrigel cultures were generated by placing Matrigel (BD; 356231) into the wells of a 8-well chamber slide (LabTek II; 154534). The chamber slide was incubated at room temperature for $15 \mathrm{~min} .2,500$ cells in a $2 \%$ Matrigel and $98 \%$ growth medium solution were seeded into each well. The chamber slide was incubated at room temperature for 30 min and then growth media was added. Cells were incubated for 8-10 days or until acini formed. Growth medium was changed every 3 days ${ }^{19}$.

\section{Stiff/Soft Substrate Culture}

Stiff substrates were generated by coating glass cover slips with bovine collagen type I (PureCol, $2.5 \mathrm{mg} / \mathrm{mL}$, Advanced BioMatrix). Collagen was diluted to $50 \mu \mathrm{g} / \mathrm{mL}$ in PBS and incubated on cover slips for $1 \mathrm{~h}$ at $37{ }^{\circ} \mathrm{C} .5 \times 10^{5}$ were plated ${ }^{14}$. Soft substrates were generated by polymerizing a bovine collagen type I gel (PureCol, $2.5 \mathrm{mg} / \mathrm{mL}$; Advanced BioMatrix) on glass cover slips. A $2 \mathrm{mg} / \mathrm{mL}$ collagen gel solution was made by mixing 8 parts of chilled bovine collagen type I (PureCol, $2.5 \mathrm{mg} / \mathrm{mL}$, Advanced BioMatrix) with 1 part of 10x PBS and 1 part of $\mathrm{NaOH}(0.1 \mathrm{~N})$. The mixture was added to the cover slips and incubated for $1 \mathrm{~h}$ at $37^{\circ} \mathrm{C}$. Cells were then seeded at $5 \times 10^{5}$ cells in $500 \mu \mathrm{L}$ of culture medium on top of the gels ${ }^{14}$. For both soft and stiff matrices, cell culture growth medium was changed every two days and cells were grown to confluence.

\section{Glucose Uptake}

Glucose uptake was measured using a kit from Cayman Chemicals (600470) and used as previously described ${ }^{11}$. For all lysates, $100 \mu \mathrm{L}$ were loaded in triplicate onto a 96-well plate and a fluorescence reading of 485/535 was taken (Biotek Synergy Neo model NEOALHPA $\mathrm{B}$, Gen 5 software). The concentration of glucose was determined using a standard curve and results are reported as micrograms per milliliter per $10^{5}$ cells.

\section{ATP Assays}

Intracellular ATP was measured using a Fluorometric ATP assay kit from Abcam (ab83355) and used as previously described ${ }^{11} .5 \mu \mathrm{L}$ of de-proteinated sample was diluted with $45 \mu \mathrm{L}$ of 
ATP assay buffer and added to ATP reaction mix in 96-well plates and fluorescence reading at $535 / 587 \mathrm{~nm}$ was made.

\section{Immunoprecipitation and immunoblotting}

For immunoprecipitation of E-cadherin or ankyrin G, cells were lysed with EB lysis buffer (1 mM Tris-HCl, pH 7.6, $50 \mathrm{mM} \mathrm{NaCl}, 1 \%$ Triton X-100, 5 mM EDTA, 50 mM NaF, 20 $\mu \mathrm{g} / \mathrm{mL}$ aprotinin, $2 \mathrm{mM} \mathrm{Na}_{3} \mathrm{VO}_{4}$, and $1 \mathrm{mM}$ PMSF). The lysates were centrifuged at 12,000 $\mathrm{xg}$ and the supernatant was collected. A 1:100 dilution of E-cadherin antibodies, HECD1 (Invitrogen) or rr1 (DSHB), or an ankyrin G antibody (h-4; Santa Cruz,) was incubated with the supernatant. The complexes were recovered using protein $\mathrm{G}$ beads, washed 3 times, and resuspended in 2x sample buffer (200 mM Tris, $\mathrm{pH} 6.8,20 \%$ glycerol, $5 \%$ BME (B-mercaptoethanol), 4\% SDS, $15 \mathrm{mg}$ Bromophenol Blue, brought up to $50 \mathrm{mLs}$ with $\mathrm{ddH}_{2} \mathrm{O}$ ) and resolved using SDS-PAGE, and transferred to PVDF (Immobilon). Samples blotted for GLUT1 were not boiled rather they were gently heated at $56{ }^{\circ} \mathrm{C}$. The membranes were blocked in 5\% BSA for pCRKL, pAMPK, AMPK, 1\% BSA for GLUT1, GLUT3, GLUT4, ankyrin G), or 5\% milk for E-cadherin, Vinculin, CRKL, B-actin. The membranes were incubated with the following antibodies $\mathrm{O} / \mathrm{N}$ at $4{ }^{\circ} \mathrm{C}$. Primary antibodies used for immunoblotting were polyclonal GLUT1 at 1:500 (abcam;15309), monoclonal E-cadherin at 1:1000 (BD; 610181), monoclonal vinculin at 1:1000 (Sigma; hVin1), monoclonal GLUT3 at 1:500 (Santa Cruz; 74399), monoclonal ankyrin G at 1:500 (Santa Cruz; H-4), at 1:1000, polyclonal phospho-CRKL at 1:1000 (Cell Signaling; 3181), monoclonal CRKL at 1:250 (Santa Cruz; C-20), polyclonal pAMPK at 1:1000 (Cell Signaling; 2535), polyclonal AMPK (Cell Signaling, 2532), and $\beta$-actin at 1:1000 (Cell Signaling, 3700). The membranes were washed $3 x$ in TBST and incubated with 1:1000 concentration of anti-mouse HRP (Jackson Labs) or anti-rabbit HRP (Jackson Labs) The membranes were washed 3x in TBST and visualized by using chemiluminescence detection reagents (Pierce), and the signal was detected on a GE Image Quant LAS 400 Imager or an Odyssey Fc Imager (Li-Cor Biosciences). For analysis, the integrated density of each band was corrected for background. Ratios of phosphorylated compared to total proteins were quantified by stripping and re-probing membranes. Quantification of each assay represents a minimum of three experiments \pm SEM. Quantification was done using either Image J (Version 2.0.1/1.53c) or Image studio lite (Version 5.2.5). Statistical analysis was conducted using two-tailed, unpaired Student's $t$-tests.

\section{Immunofluorescence of 2D Cell Culture}

Coverslips were coated with human fibronectin $(10 \mu \mathrm{g} / \mathrm{mL})$, and cells were plated and grown to confluency. Cells were fixed with $4 \%$ paraformaldehyde, permeabilized with $0.5 \%$ triton and washed with either universal buffer (UB; $150 \mathrm{mM} \mathrm{NaCl}, 50 \mathrm{mM}$ Tris, $\mathrm{pH}$ 7.6, and $0.01 \% \mathrm{NaN} 3$ ) or PBS. Cells were blocked with either $10 \%$ bovine serum albumin (Sigma) in UB or 5\% BSA and 1\% goat serum in PBS depending on primary antibody. Antibodies used: GLUT1 monoclonal antibody at 1:200 (Abcam), polyclonal $\beta$-catenin antibody at 1:500 (Sigma), monoclonal E-cadherin antibodies, HECD1 at 1:200 (Invitrogen) and rr1 at 1:50 (DSHB), monoclonal ankyrin G antibody at 1:200 (Santa Cruz), monoclonal $\beta$-spectrin antibody at 1:300 (BD Transduction, 612563), DAPI Solution at 1:5,000 (BD Pharmigen, 564907) and F-actin was stained using phalloidin conjugated with alexa-594 
(1:300). Secondary antibodies used were anti-mouse alexa-488, anti-rabbit alexa-594, antimouse alexa-594 or anti-mouse alexa-647, 1:400 in blocking solution.

\section{Immunofluorescence of 3D Cell Culture}

Matrigel embedded cells were fixed in 4\% PFA in 1x PBS permeabilized using $0.2 \%$ Triton X-100 in PBS, and washed $3 \mathrm{x}$ with PBS $+100 \mathrm{mM}$ glycine. Cells were blocked for 90 minutes at room temperature in blocking buffer $\left(7.7 \mathrm{mM} \mathrm{NaN}_{3}, 0.1 \%\right.$ BSA, 0.2 $\%$ Triton X-100, 0.05\% Tween-20, 10\% goat serum in 1x PBS). Primary antibodies were diluted in the blocking solution incubated with the cells $\mathrm{O} / \mathrm{N}$ at $4{ }^{\circ} \mathrm{C}$. Cells were washed $3 \mathrm{x}$ with washing buffer (blocking buffer minus the goat serum). Secondary antibodies were diluted in the blocking solution and incubated with the cells for 1 hours at RT. Cells were then washed 2x with washing buffer (blocking buffer minus goat serum), 1x with PBS + DAPI , and then $1 \mathrm{x}$ with PBS. E-cadherin was stained using a monoclonal antibody, $\operatorname{rr} 1$ at 1:50 (DSHB). GLUT1 was stained using a polyclonal antibody at 1:300 (Abcam, 15309). Secondary antibodies used were anti-mouse alexa-647 (Invitrogen, A-21235), and anti-rabbit alexa-488 (Invitrogen, A-11008) in blocking solution.

\section{Quantification of Immunofluorescence Images}

Fluorescence images were captured at room temperature with a confocal microscope (model LSM 710; Carl Zeiss Micro Imaging, Inc.). A 40× oil objective (Carl Zeiss Micro Imaging, Inc.) with a numerical aperture of 1.3 was used. Images were obtained using the Zen2009 software (Carl Zeiss Micro Imaging, Inc.). Laser settings were kept identical for all conditions for each individual experiment. Quantifications of images were made using ImageJ. Indicated number of junctions were chosen at random over at least five fields of view. Individual junctions were outlined creating unique regions of interest (ROI). The integrated density was measured for each ROI and across corresponding channels captured. Non-junctional staining was measured by shifting ROI to the cytoplasmic region. Graphs report the corrected fluorescence intensity of the ROI, the corrected fluorescence intensity $=$ (integrated density of junction area - integrated density of non-junction area). Data represented as a box and whisker plots with 90-10 percentile shown. Pearson correlation coefficient was calculated by comparing two arrays of corrected fluorescence intensities for indicated number ofjunctions using the following equation: $\mathrm{r}=\frac{\sum(x-\bar{x})(y-\bar{y})}{\sqrt{\sum(x-\bar{x})^{2} \sum(y-\bar{y})^{2}}}$

\section{Transepithelial Resistance Assay}

Cells were plated at a density of $5 \times 10^{4}$ cells per well of a Costar® $0.4 \mu \mathrm{m}$ Polycarbonate

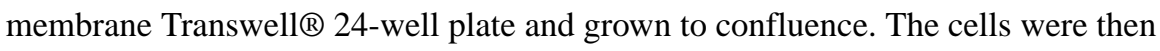
incubated in calcium free DMEM overnight. Growth media was added back to the cultures for the indicated times and transepithelial electrical resistance was measured in triplicate using a Millipore Voltmeter (MERS 000 01). Results are in $\Omega * \mathrm{~cm}^{2}$.

\section{Statistics and Reproducibility}

Statistical differences between groups of data were analyzed using a series of two-tailed unpaired Student t-tests. All statistical analysis and data graphing was done on Prism 
(Version 9.0.1). All quantified experiments represent at least three biologically independent samples with the exception of immunofluorescence data. All immunofluorescence experiments were repeated independently three times with similar results.

\section{Data Availability}

All data supporting the findings of this study are available from the corresponding author upon reasonable request.

\section{Code Availability}

No collection software was used in this study. Source data are provided with this paper. 


\section{Extended Data}
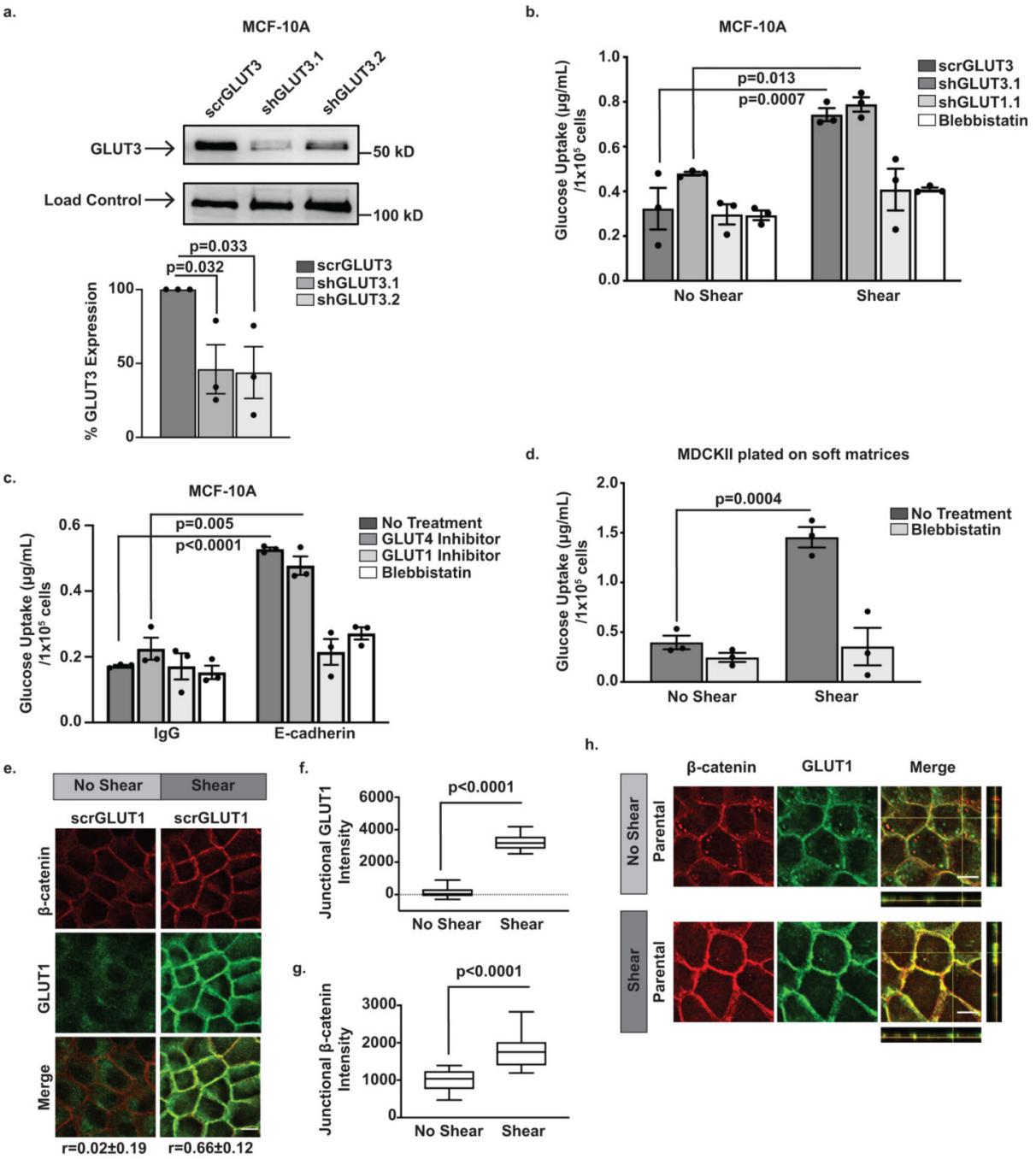

h.
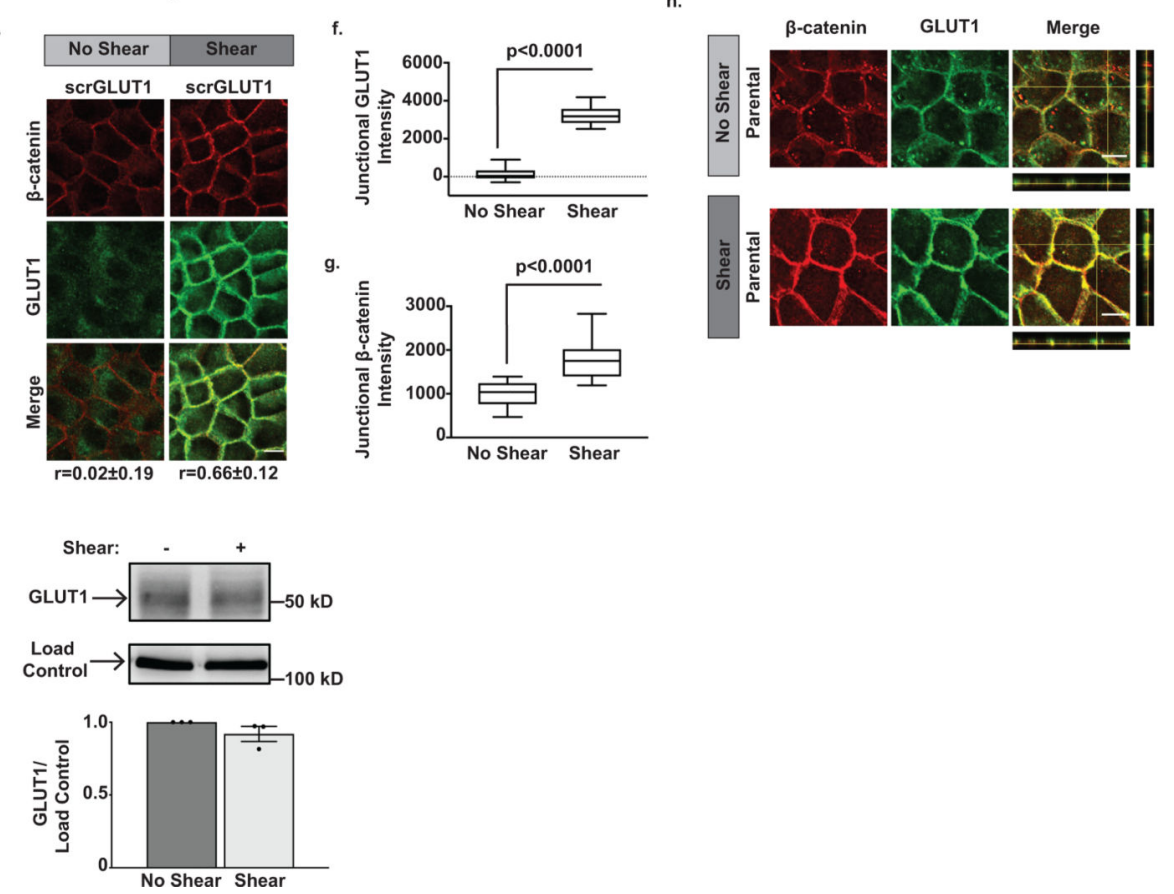

Extended Data Figure 1:

GLUT1 mediates force-induced glucose uptake. 

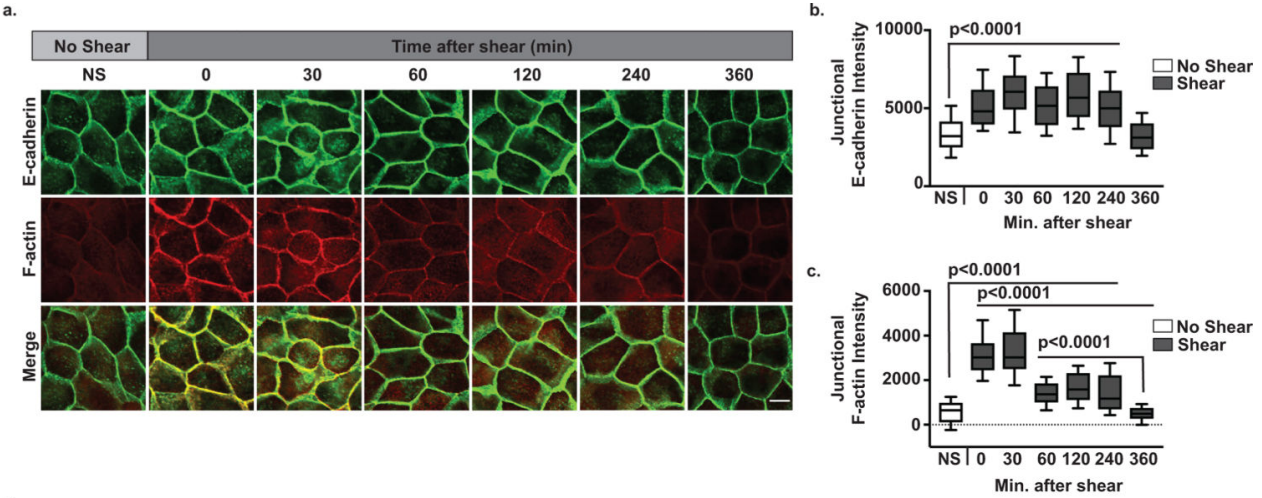

d.

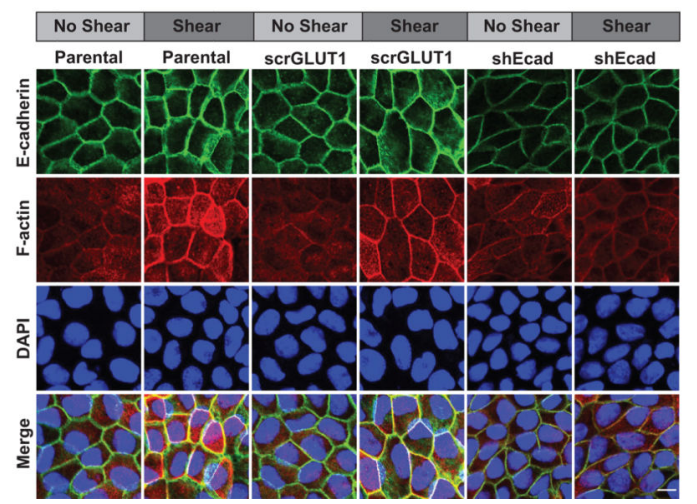

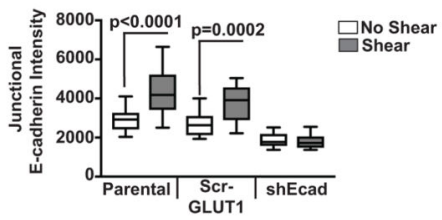

.

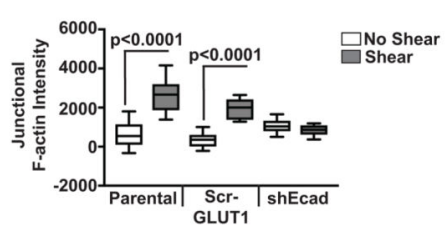

Extended Data Figure 2:

Cell stiffening is a transient process that can be visualized by staining cells with F-actin and E-cadherin. 
a.
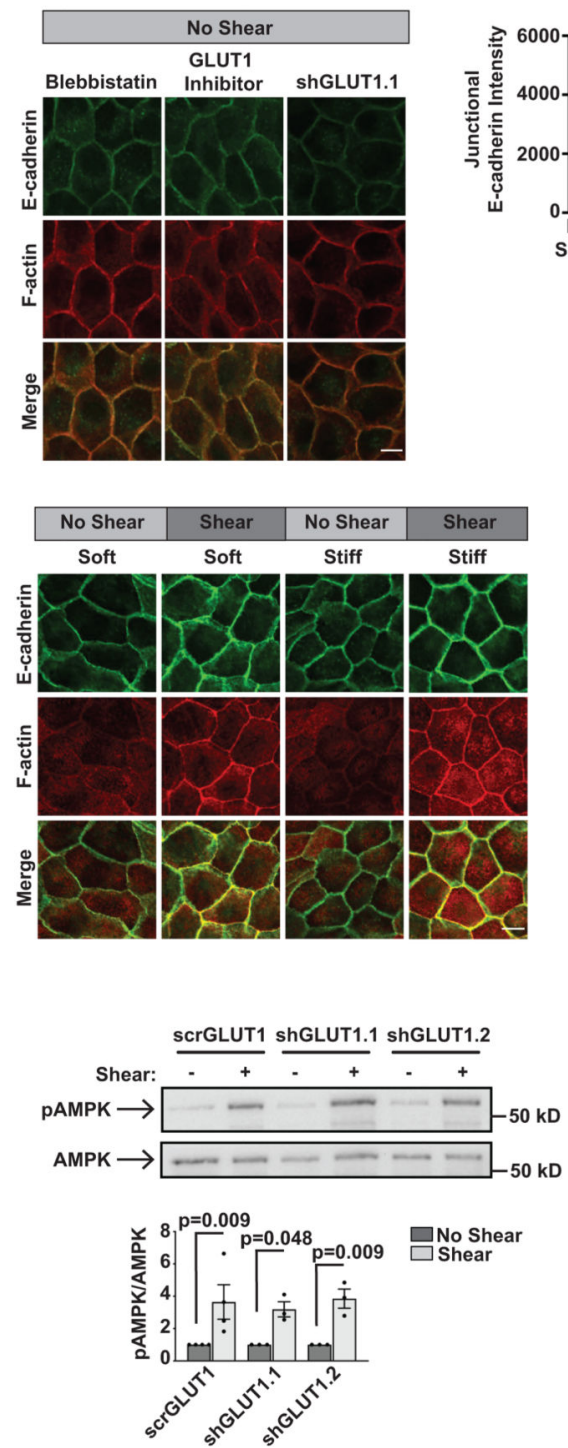

h.

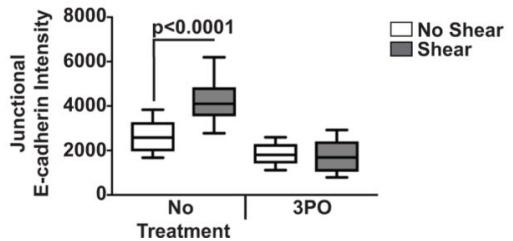

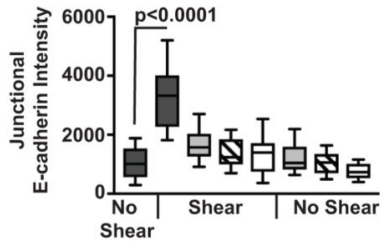

Parental

GLUT1 Inhibito

shGLUT1.1

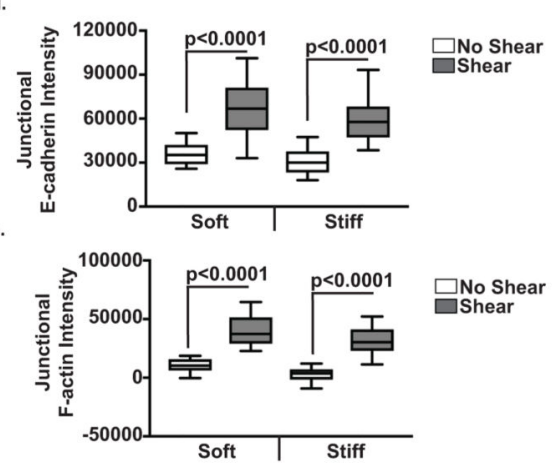

g.
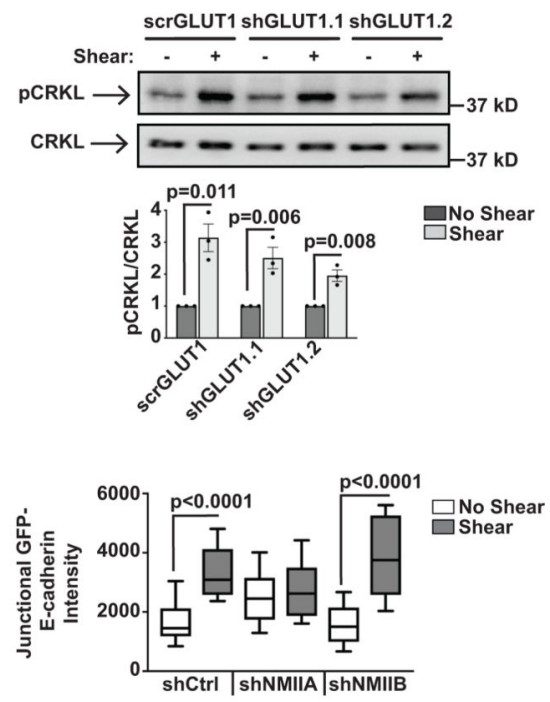

Extended Data Figure 3:

Control studies for the effects of shear on reinforment of the actin cytoskeleton. 
a.

d.
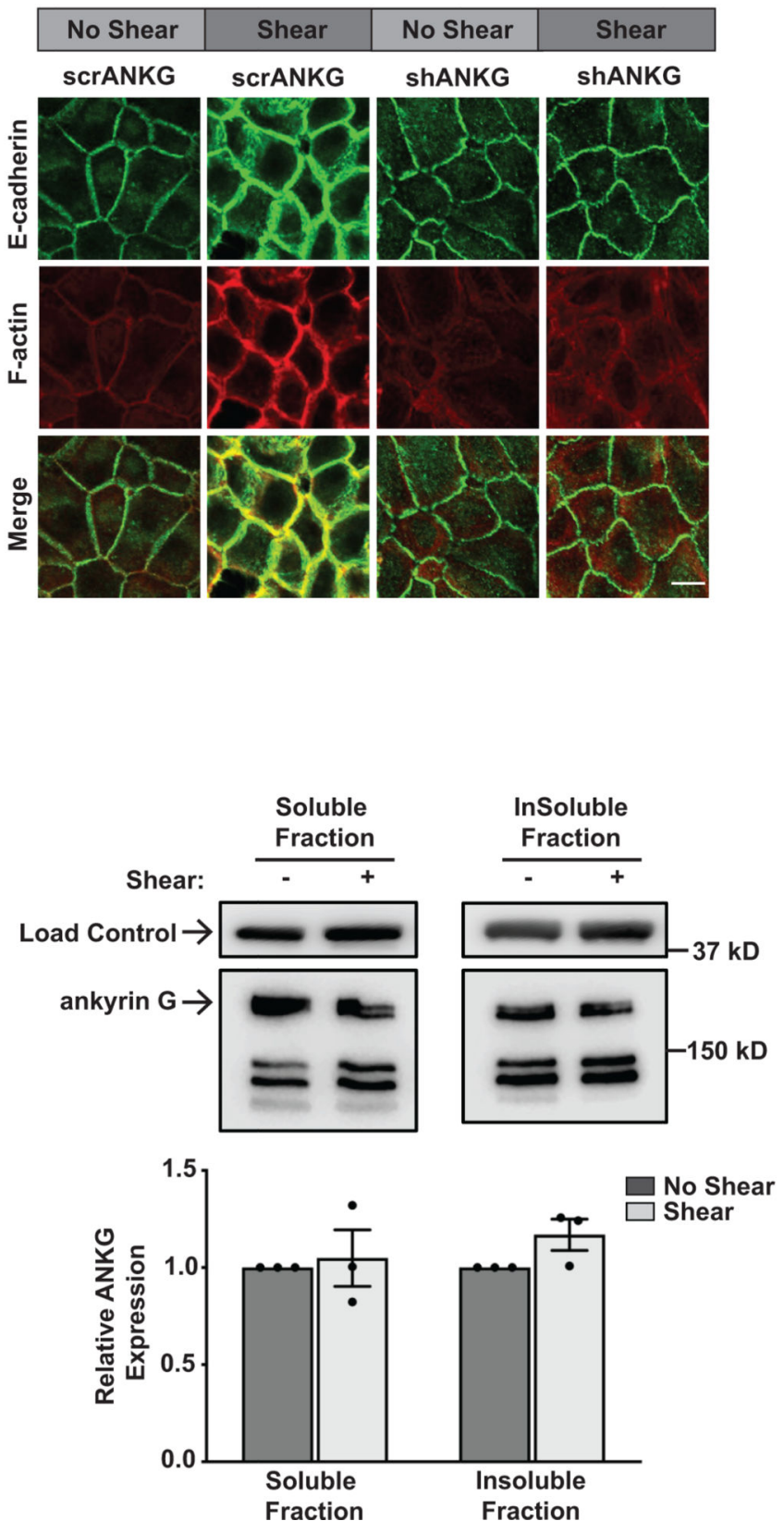

Extended Data Figure 4:

Ankyrin G mediates GLUT1 and E-cadherin complex. b.

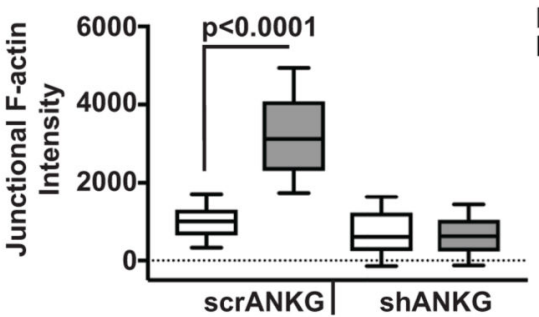

c.

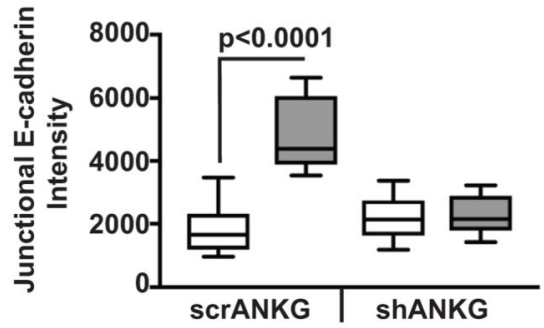

e.

\begin{tabular}{|c|c|}
\hline No Shear & Shear \\
\hline Parental & Parental \\
\hline
\end{tabular}
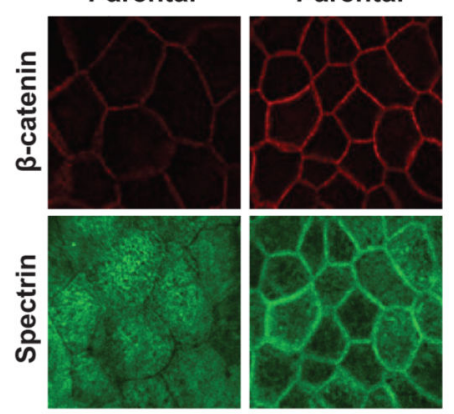

$\frac{\stackrel{0}{\circ}}{\Sigma}$

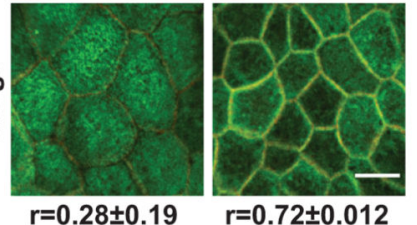

No Shear

Shear

No Shear Shear 
a.
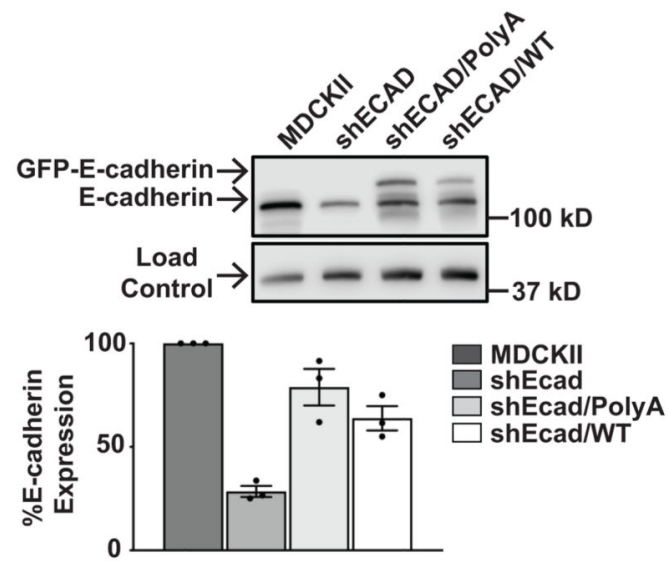

c.
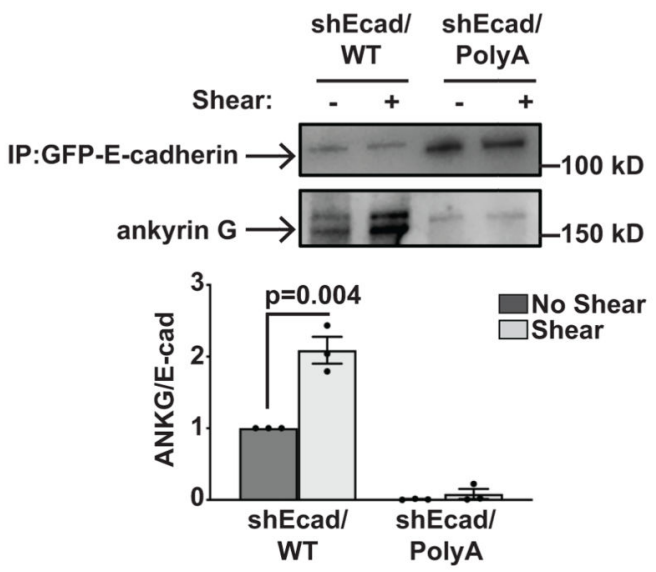

b.

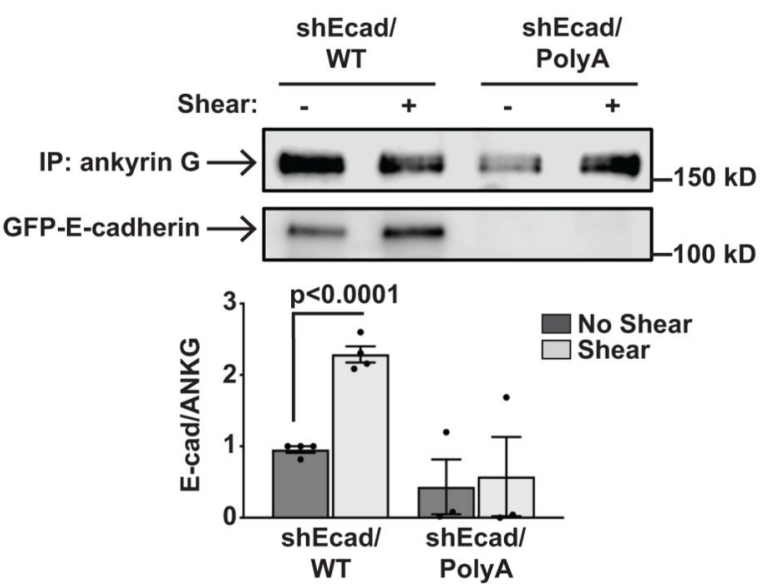

d.
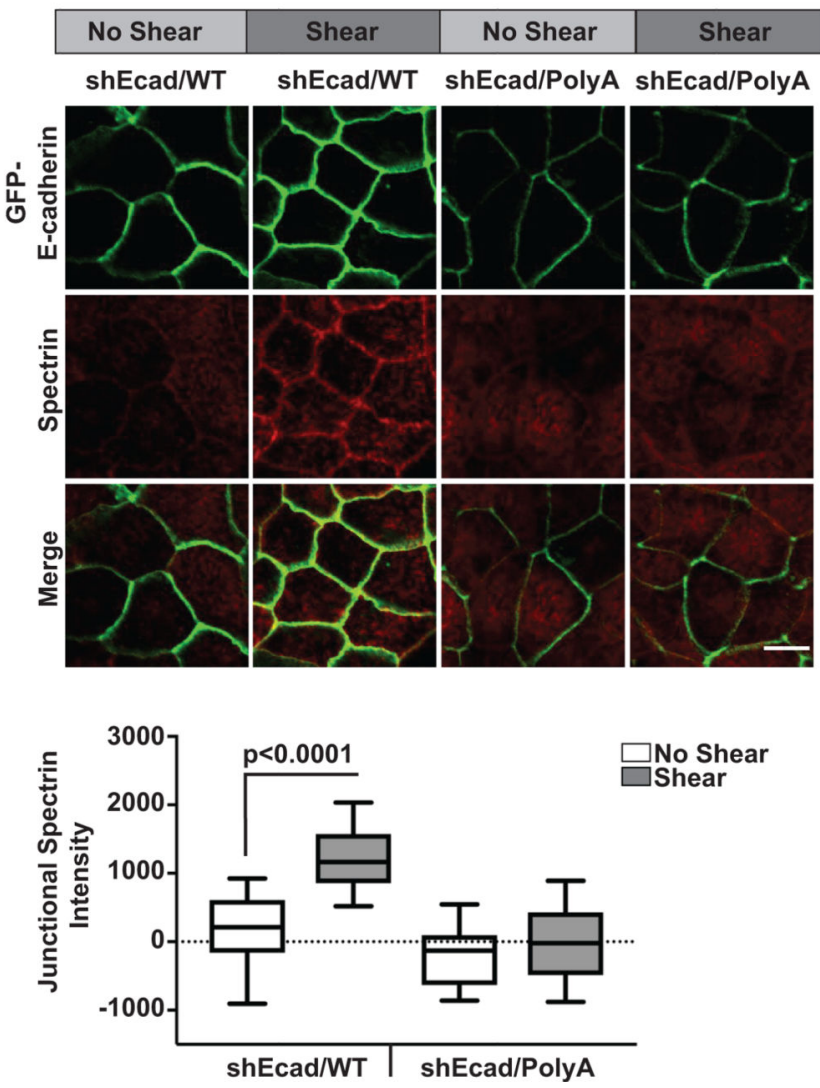

Extended Data Figure 5:

Ankyrin G mediates a force-induced complex between E-cadherin and GLUT1.

\section{Acknowledgements:}

We thank Peter Rubenstein and Gillian DeWane for comments. This work is supported by National Institutes of Health Grants \#R35GM136291 to KAD and P30CA086862 to the Holden Comprehensive Cancer Center. RMM was supported by Agence Nationale de la Recherche "POLCAM" (ANR-17- CE13-0013), "CODECIDE" (ANR-17-CE-13-0022) Ligue Contre le Cancer (Equipe labellisée 2019). National Institutes of Health predoctoral fellowships \#T32 GM067795 and \#1F31GM135962-01 supported AMS. American Heart Association Grant \#16PRE26701111 supported JLB. 


\section{References:}

1. Fletcher DA \& Mullins RD Cell mechanics and the cytoskeleton. Nature 463, 485-492 (2010). [PubMed: 20110992]

2. Bernstein BW \& Bamburg JR Actin-ATP hydrolysis is a major energy drain for neurons. J Neurosci 23, 1-6 (2003). [PubMed: 12514193]

3. Daniel JL, Molish IR, Robkin L \& Holmsen H. Nucleotide exchange between cytosolic ATP and F-actin-bound ADP may be a major energy-utilizing process in unstimulated platelets. Eur J Biochem 156, 677-684 (1986). [PubMed: 3699030]

4. Ferrell N. et al.Application of physiological shear stress to renal tubular epithelial cells. Methods Cell Biol153, 43-67 (2019). [PubMed: 31395384]

5. Neville MCClassic studies of mammary development and milk secretion: 1945 - 1980. J Mammary Gland Biol Neoplasia14, 193-197 (2009). [PubMed: 19657595]

6. Guilluy C. et al.The Rho GEFs LARG and GEF-H1 regulate the mechanical response to force on integrins. Nat Cell Biol13, 722-727 (2011). [PubMed: 21572419]

7. Marjoram RJ, Guilluy C \& Burridge K. Using magnets and magnetic beads to dissect signaling pathways activated by mechanical tension applied to cells. Methods 94, 19-26 (2016). [PubMed: 26427549]

8. Barry AKet al.alpha-catenin cytomechanics--role in cadherin-dependent adhesion and mechanotransduction. J Cell Sci127, 1779-1791 (2014). [PubMed: 24522187]

9. Collins C. et al.Localized tensional forces on PECAM-1 elicit a global mechanotransduction response via the integrin-RhoA pathway. Curr Biol22, 2087-2094 (2012). [PubMed: 23084990]

10. Kim TJet al.Dynamic visualization of alpha-catenin reveals rapid, reversible conformation switching between tension states. Curr Biol25, 218-224 (2015). [PubMed: 25544608]

11. Bays JL, Campbell HK, Heidema C, Sebbagh M \& DeMali KA Linking E-cadherin mechanotransduction to cell metabolism through force-mediated activation of AMPK. Nat Cell Biol 19, 724-731 (2017). [PubMed: 28553939]

12. Bays JLet al.Vinculin phosphorylation differentially regulates mechanotransduction at cell-cell and cell-matrix adhesions. J Cell Biol205, 251-263 (2014). [PubMed: 24751539]

13. Tzima E. et al.A mechanosensory complex that mediates the endothelial cell response to fluid shear stress. Nature437, 426-431 (2005). [PubMed: 16163360]

14. Park JSet al.Mechanical regulation of glycolysis via cytoskeleton architecture. Nature578, 621-626 (2020). [PubMed: 32051585]

15. Camps M, Vilaro S, Testar X, Palacin M \& Zorzano A. High and polarized expression of GLUT1 glucose transporters in epithelial cells from mammary gland: acute down-regulation of GLUT1 carriers by weaning. Endocrinology 134, 924-934 (1994). [PubMed: 8299587]

16. Yang Q. et al.PRKAA1/AMPKalpha1-driven glycolysis in endothelial cells exposed to disturbed flow protects against atherosclerosis. Nat Commun9, 4667 (2018). [PubMed: 30405100]

17. Farrell CL, Yang J \& Pardridge WM GLUT-1 glucose transporter is present within apical and basolateral membranes of brain epithelial interfaces and in microvascular endothelia with and without tight junctions. J Histochem Cytochem 40, 193-199 (1992). [PubMed: 1552163]

18. Capaldo CT \& Macara IG Depletion of E-cadherin disrupts establishment but not maintenance of cell junctions in Madin-Darby canine kidney epithelial cells. Mol Biol Cell 18, 189-200 (2007). [PubMed: 17093058]

19. O’Brien LE, Zegers MM \& Mostov KE Opinion: Building epithelial architecture: insights from three-dimensional culture models. Nat Rev Mol Cell Biol 3, 531-537 (2002). [PubMed: 12094219]

20. Yu W. et al.Formation of cysts by alveolar type II cells in three-dimensional culture reveals a novel mechanism for epithelial morphogenesis. Mol Biol Cell18, 1693-1700 (2007). [PubMed: 17332496]

21. Zegers MM, O'Brien LE, Yu W, Datta A \& Mostov KE Epithelial polarity and tubulogenesis in vitro. Trends Cell Biol 13, 169-176 (2003). [PubMed: 12667754] 
22. Heuze MLet al.Myosin II isoforms play distinct roles in adherens junction biogenesis. Elife8 (2019).

23. Bennett V \& Healy J. Membrane domains based on ankyrin and spectrin associated with cell-cell interactions. Cold Spring Harb Perspect Biol 1, a003012 (2009).

24. Nelson WJ, Shore EM, Wang AZ \& Hammerton RW Identification of a membrane-cytoskeletal complex containing the cell adhesion molecule uvomorulin (E-cadherin), ankyrin, and fodrin in Madin-Darby canine kidney epithelial cells. J Cell Biol 110, 349-357 (1990). [PubMed: 2153683]

25. Kizhatil K. et al.Ankyrin-G is a molecular partner of E-cadherin in epithelial cells and early embryos. J Biol Chem282, 26552-26561 (2007). [PubMed: 17620337]

26. Jenkins PMet al.E-cadherin polarity is determined by a multifunction motif mediating lateral membrane retention through ankyrin-G and apical-lateral transcytosis through clathrin. J Biol Chem288, 14018-14031 (2013). [PubMed: 23530049]

27. Cadwell CM, Jenkins PM, Bennett V \& Kowalczyk AP Ankyrin-G Inhibits Endocytosis of Cadherin Dimers. J Biol Chem 291, 691-704 (2016). [PubMed: 26574545]

28. Yang HQet al.Ankyrin-G mediates targeting of both $\mathrm{Na}(+)$ and KATP channels to the rat cardiac intercalated disc. Elife9 (2020).

29. Zhou D. et al.AnkyrinG is required for clustering of voltage-gated $\mathrm{Na}$ channels at axon initial segments and for normal action potential firing. J Cell Biol143, 1295-1304 (1998). [PubMed: 9832557]

30. Lowe JSet al.Voltage-gated Nav channel targeting in the heart requires an ankyrin-G dependent cellular pathway. J Cell Biol180, 173-186 (2008). [PubMed: 18180363]

31. Baines AJThe spectrin-ankyrin-4.1-adducin membrane skeleton: adapting eukaryotic cells to the demands of animal life. Protoplasma244, 99-131 (2010). [PubMed: 20668894]

32. Bennett V. The molecular basis for membrane - cytoskeleton association in human erythrocytes. $\mathrm{J}$ Cell Biochem18, 49-65 (1982). [PubMed: 6461664]

33. Drenckhahn D, Schluter K, Allen DP \& Bennett V. Colocalization of band 3 with ankyrin and spectrin at the basal membrane of intercalated cells in the rat kidney. Science 230, 1287-1289 (1985). [PubMed: 2933809]

34. Drenckhahn D \& Bennett V. Polarized distribution of Mr 210,000 and 190,000 analogs of erythrocyte ankyrin along the plasma membrane of transporting epithelia, neurons and photoreceptors. Eur J Cell Biol 43, 479-486 (1987). [PubMed: 2957198]

35. Khan AAet al.Dematin and adducin provide a novel link between the spectrin cytoskeleton and human erythrocyte membrane by directly interacting with glucose transporter-1. J Biol Chem283, 14600-14609 (2008). [PubMed: 18347014]

36. Jiang W. et al.Interaction of glucose transporter 1 with anion exchanger 1 in vitro. Biochem Biophys Res Commun339, 1255-1261 (2006). [PubMed: 16343432]

37. Zhang J. et al.Energetic regulation of coordinated leader-follower dynamics during collective invasion of breast cancer cells. Proc Natl Acad Sci U S A116, 7867-7872 (2019). [PubMed: 30923113]

38. DeCamp SJet al.Epithelial layer unjamming shifts energy metabolism toward glycolysis. Sci Rep10, 18302 (2020). [PubMed: 33110128]

39. Zanotelli MRet al.Energetic costs regulated by cell mechanics and confinement are predictive of migration path during decision-making. Nat Commun10, 4185 (2019). [PubMed: 31519914]

40. Kannan N \& Tang VW Synaptopodin couples epithelial contractility to alpha-actinin-4-dependent junction maturation. J Cell Biol 211, 407-434 (2015). [PubMed: 26504173]

41. Maiers JL, Peng X, Fanning AS \& DeMali KA ZO-1 recruitment to alpha-catenin--a novel mechanism for coupling the assembly of tight junctions to adherens junctions. J Cell Sci 126, 3904-3915 (2013). [PubMed: 23813953]

42. Tang VW \& Goodenough DA Paracellular ion channel at the tight junction. Biophys J 84, 1660 1673 (2003). [PubMed: 12609869]

43. Peng X, Cuff LE, Lawton CD \& DeMali KA Vinculin regulates cell-surface E-cadherin expression by binding to beta-catenin. J Cell Sci 123, 567-577 (2010). [PubMed: 20086044] 


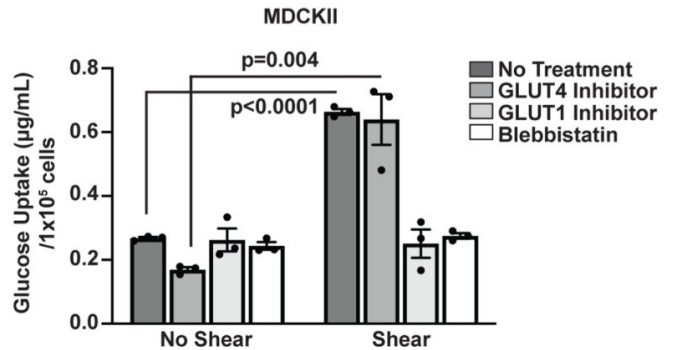

c.
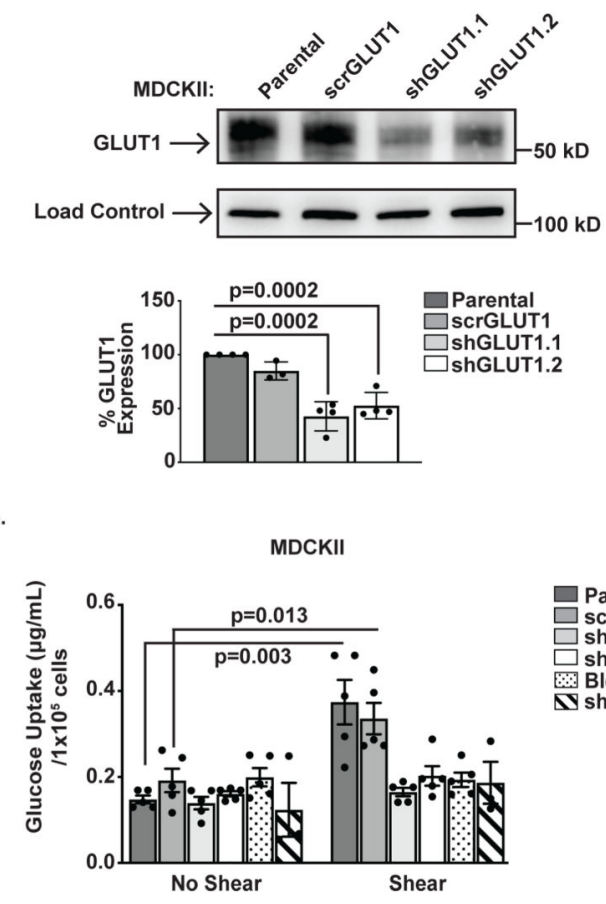

g

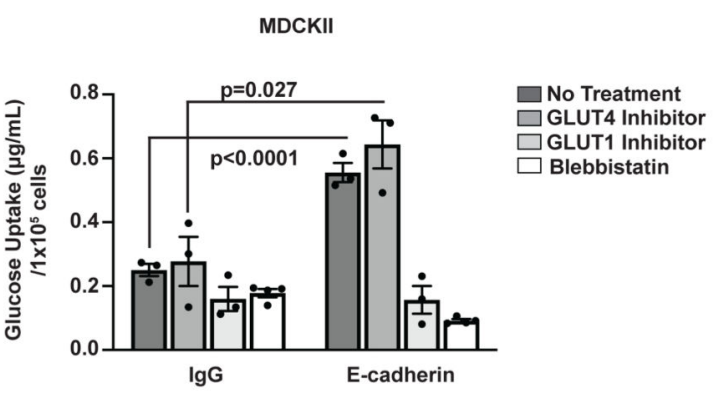

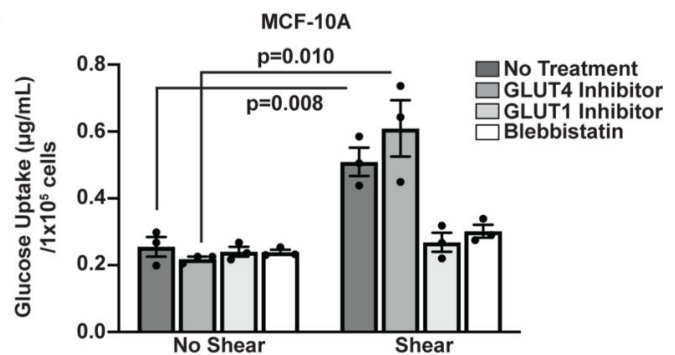

d.

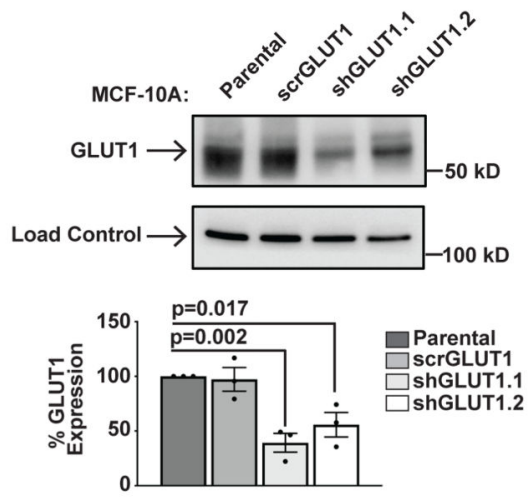

f.

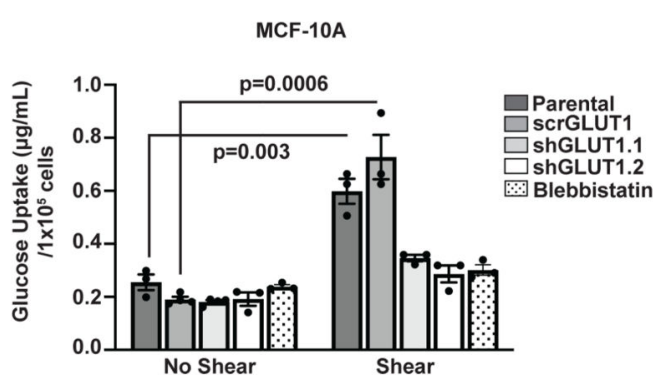

h.

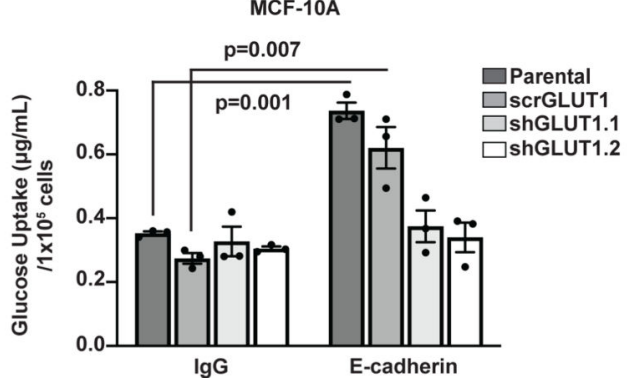

Figure 1: GLUT1 mediates force-induced glucose uptake.

( $a$ and b) Pre-incubation of cells with GLUT1 inhibitors prevented glucose uptake. MDCKII (a) or MCF-10A (b) cells were incubated with the indicated inhibitors and the cells were left resting (no shear) or subjected to orbital shear stress for 2 hours (shear stress). Cells were lysed and the amount of a fluorescently labelled glucose analog, 2-deoxyglucose, taken up into the cells was monitored using a fluorimeter. The graphs represent the average glucose taken up into the cells \pm SEM, $n=3$ biologically independent samples. (c and d), GLUT1 was stably inhibited using RNAi. MDCKII (c) or MCF-10A (d) cells were infected 
with retroviruses encoding shRNAs targeting two distinct sites on GLUT1 (shGLUT1.1, shGLUT1.2) or a scramble sequence (scrGLUT1). GLUT1 expression was examined by immunoblotting whole cell lysates with an antibody against GLUT1 or vinculin as a loading control. The graphs beneath the blots depict the average amount of GLUT1 in the cells expressed as function of total GLUT1 levels in the parental cells \pm SEM, $n=3$ biologically independent samples (e-h) Inhibition of GLUT1 using RNA interference or pharmacological inhibitor prevented glucose uptake in response to application of shear stress to MDCKII (e) or MCF-10A (f) or application of tensile forces to MDCKII (g) or MCF-10A cells (h). Shear stress was applied as described in (a) or tensile forces were applied to magnetic beads coated with E-cadherin extracellular domains (E-cadherin) or IgG as a control. A $10 \mathrm{pN}$ tensile force was applied for 10 minutes. Glucose uptake was monitored as described above. The graphs represent average glucose taken up into the cells \pm SEM. (g) $n=5$ (MDCKII), $n=4$ (GLUT4 inhibitor), $n=3$ (GLUT1 inhibitor), $n=4$ (Blebbistatin). (h) $n=3$. For all experiments, significance was calculated using a two-tailed unpaired Student t-test. 

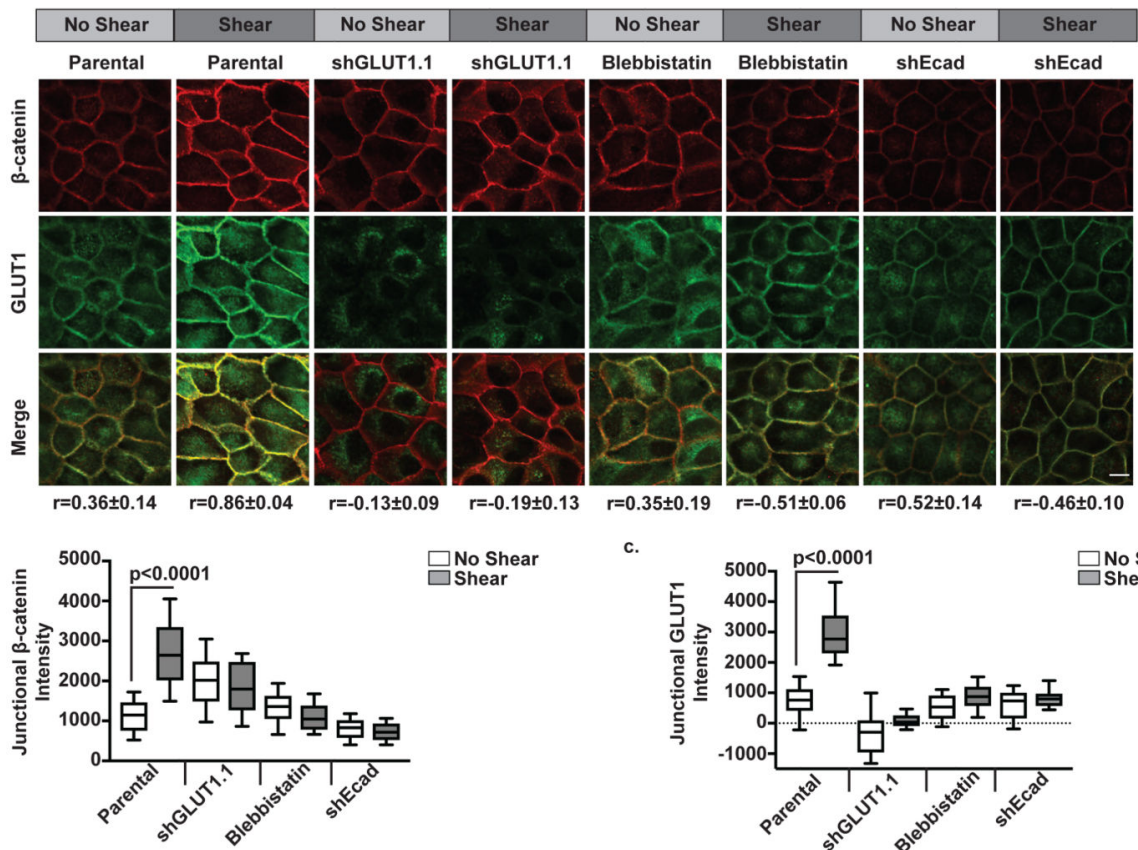

$\square$ No Shear

c.
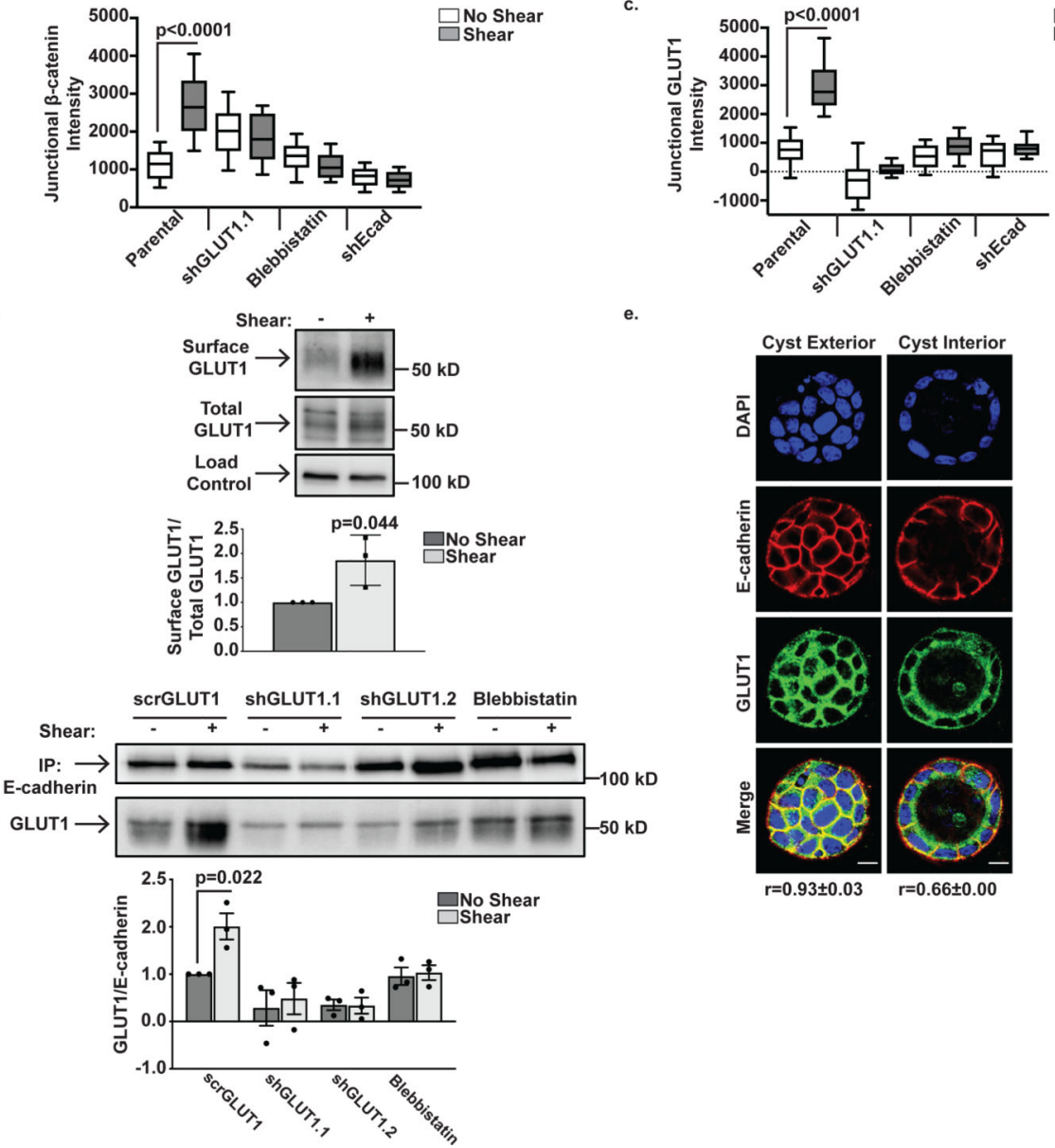

Figure 2: Force stimulates GLUT1 enrichment in cell-cell junctions.

(a-c) GLUT1 is localized to cell-cell contacts in response to application of shear stress. MDCKII or MDCKII cells with GLUT1 (shGLUT1.1 or shGLUT1.2) or E-cadherin (shEcad) stably inhibited were left resting (no shear) or exposed to shear stress for $2 \mathrm{~h}$. The cells were fixed and stained with antibodies against GLUT1 or $\beta$-catenin (a marker of cell-cell junctions) and examined by confocal microscopy using identical laser settings. Representative images are shown, and an average Pearson correlation coefficient \pm SEM is reported beneath the images. Scale bars $=10 \mu \mathrm{M}$. The graphs represent the average corrected 
fluorescence intensity of $\beta$-catenin (b) or GLUT1 (c) in 50 junctions per cell type. The data are represented as a box and whisker plot with median, 10th, 25th, 75th, and 90th percentiles shown, $n=50$ junctions over 5 fields of view. (d) Shear increases surface expression of GLUT1. MDCKII cells were either left resting (no shear) or exposed to shear stress for 2 h. The cells were put on ice, incubated with sulfo-NHS-LC-biotin, lysed and biotin labeled proteins were recovered. The biotinylated protein fraction was probed for GLUT1 and total cell lysates were probed for GLUT1 and vinculin (load control). The graph beneath the immunoblot image shows the average biotinylated GLUT1 recovered normalized to total GLUT1 levels \pm SEM, $n=3$ biologically independent samples. (e) GLUT1 co-localizes with E-cadherin in 3D matrices. MDCKII cells were embedded in 3D Matrigel and grown for 10 days. The cysts were fixed, permeabilized and stained for GLUT1, E-cadherin, and DAPI. Representative images of the hollow cyst interior and the cyst exterior are shown. Scale bars $=10 \mu \mathrm{M}$. The graph beneath the immunofluorescent images shows the average Pearson correlation coefficient between GLUT1/E-cadherin staining, $n=16-17$ junctions over 3 fields of view. (f) GLUT1 co-immunoprecipitates with E-cadherin. The indicated MDCKII cells with and without shear were lysed, and E-cadherin was immunoprecipitated. GLUT1 recovery was examined by immunoblotting. The graphs beneath each immunoblot image show the average amount of GLUT1 recovered and normalized for E- cadherin levels \pm SEM, $n=3$ biologically independent samples. For all experiments, significance was calculated using a two-tailed unpaired Student t-test. 

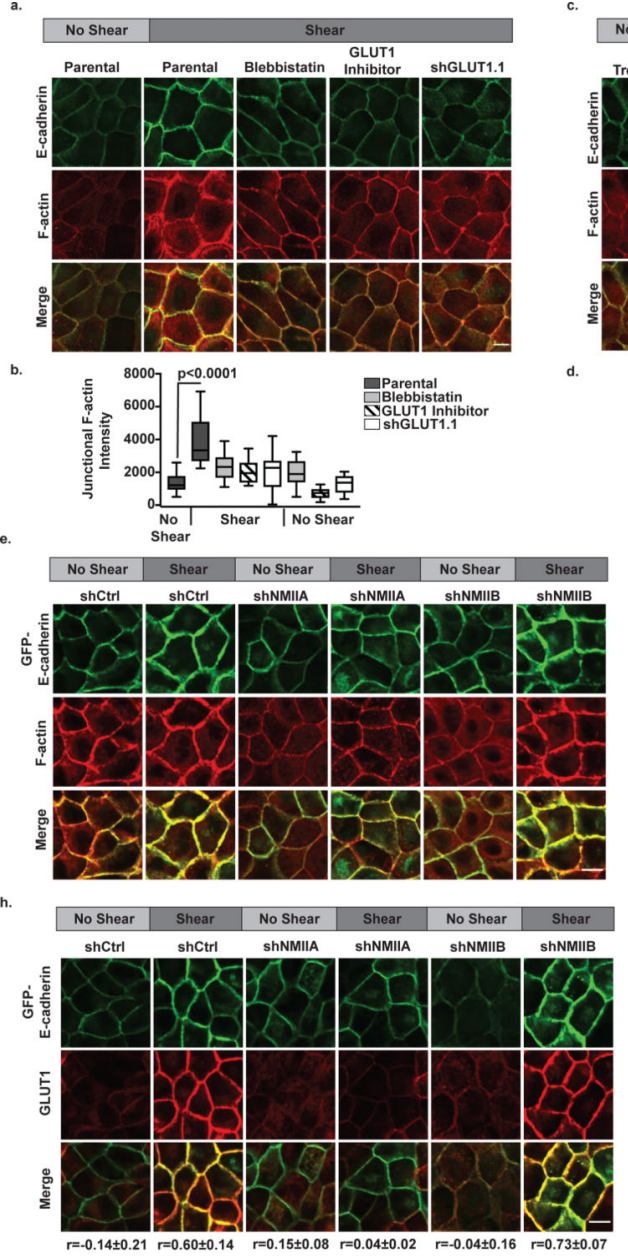
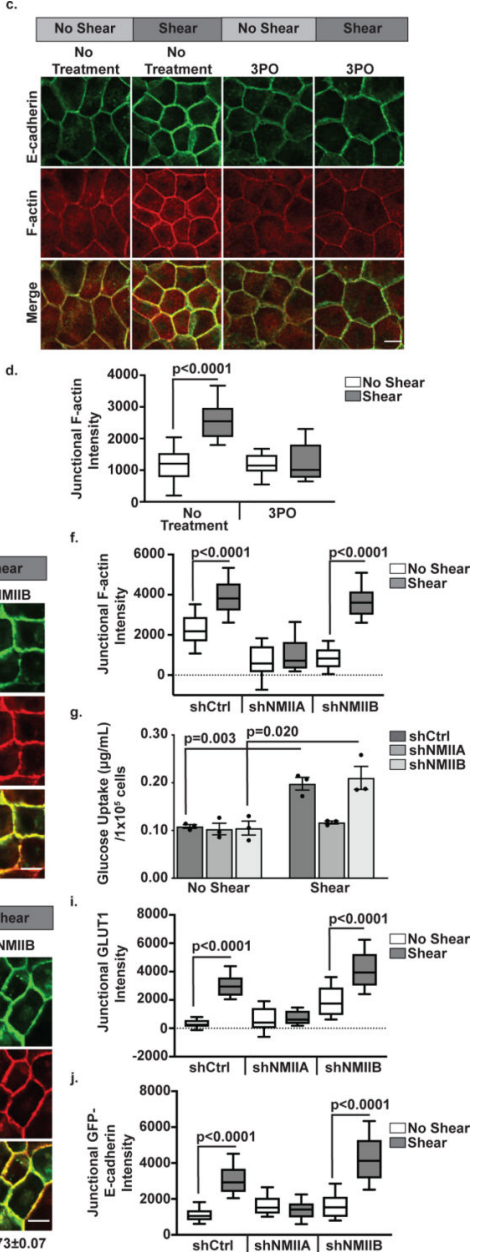

Figure 3: GLUT1 is required for non-muscle myosin IIA-dependent reinforcement of the actin cytoskeleton.

(a-d) Inhibition of GLUT1 or glycolysis prevents reinforcement of the actin cytoskeleton. MDCKII or MDCKII cells with GLUT1 stably inhibited (shGLUT1.1) were left resting (no shear) or exposed to shear stress. The cells were stained with phalloidin or antibodies against E-cadherin. Representative images are shown in a and c. Where indicated, the cells were pretreated with inhibitors against myosin II (Blebbistatin), GLUT1 (WZB117), or phosphofructokinase-2 (3PO). The graphs in $\mathrm{b}$ and $\mathrm{d}$ represent the average corrected fluorescence intensity of F-actin in junctions for the images in a and c, respectively. $\mathrm{n}=30-50$ junctions over 5 fields of view. (e and f) Non-muscle myosin IIA is required for reinforcement of the actin cytoskeleton in response to shear. MDCKII cells expressing GFP-E-cadherin were treated with either a control (shCtrl) or shRNA targeted against non-muscle myosin IIA (shNMIIA) or non-muscle myosin IIB (shNMIIB). Cells were left resting (no shear) or exposed to shear. The cells were fixed and stained with phalloidin. Representative images are shown in e. Graph $\mathrm{f}$ represents the average corrected fluorescence intensity of junctional F-actin. $\mathrm{n}=50$ junctions over 5 fields of view. (g) Non-muscle myosin IIA, but not IIB, is required for force stimulated glucose uptake. Cells were lysed, and the amount of a fluorescently labelled glucose analog, 2-deoxyglucose, taken up into the cells was monitored using a fluorimeter. The graphs represent the average glucose taken 
up into the cells \pm SEM, $n=3$ biologically independent samples. (h-j) Inhibition of NMIIA blocks GLUT1 recruitment at cell-cell contacts in response to application of shear stress. Cells were fixed and stained with an antibody against GLUT1. The graphs represent the average corrected fluorescence intensity of junctional GLUT1 (i) or GFP-E-cadherin (j). $\mathrm{n}=50$ junctions over 5 fields of view. An average Pearson correlation coefficient \pm SEM is reported beneath the images. All immunofluorescence data in this figure are represented as a box and whisker plot with median, 10th, 25th, 75th, and 90th percentiles shown. Scale bars $=10 \mu \mathrm{M}$. For all experiments, significance was calculated using a two-tailed unpaired Student t-test. 

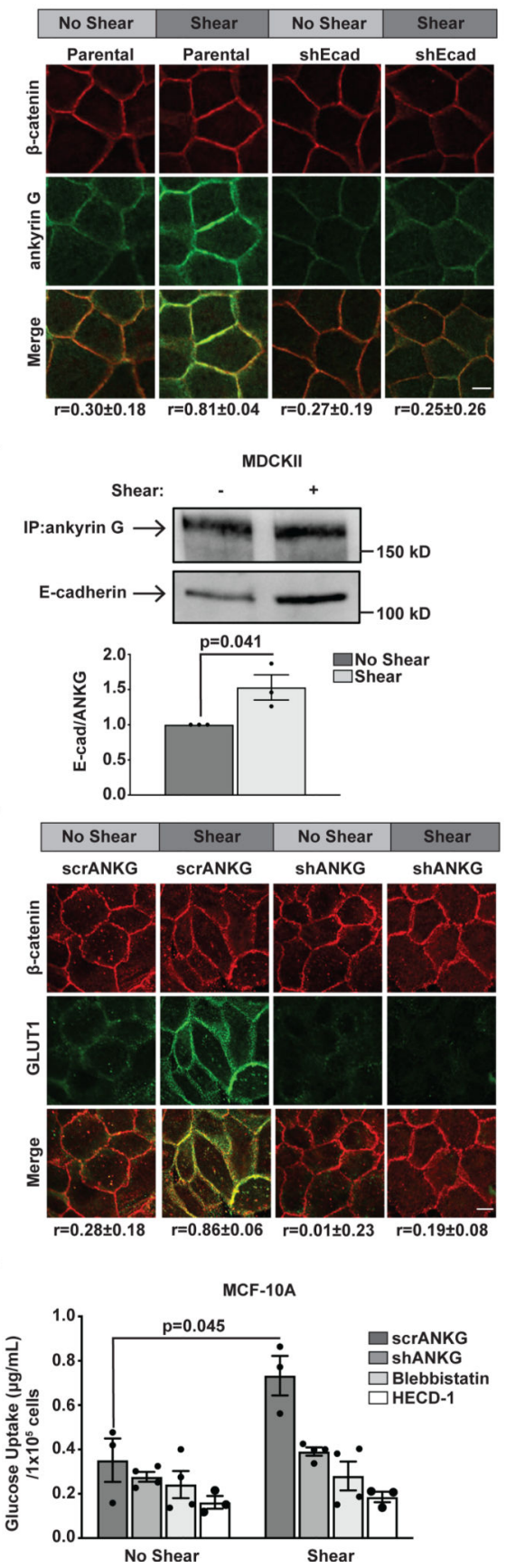
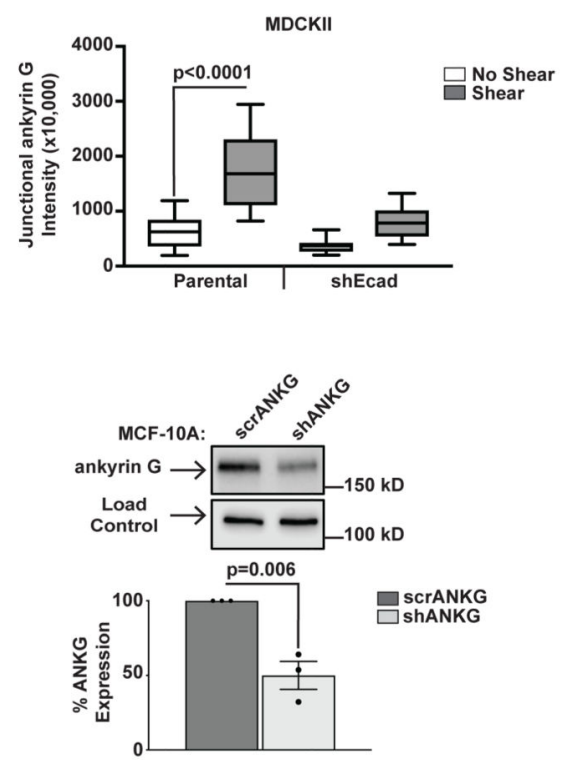

f.

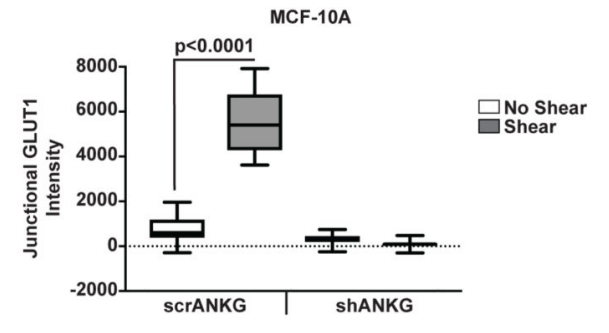

h.
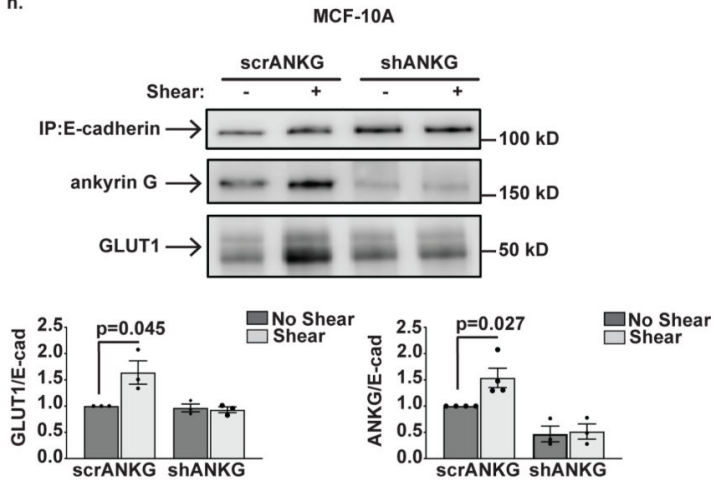

Figure 4: GLUT1 recruitment to cell-cell junctions requires ankyrin G

(a-c) Ankyrin G is recruited to cell-cell junctions and binds E-cadherin. MDCKII or MDCKII cells lacking E-cadherin (shEcad) were left resting (no shear) or exposed to shear. (a) Representative images of cells, stained with antibodies against ankyrin $\mathrm{G}$ or P-catenin, are shown. The graph in b shows the average corrected fluorescence intensity of junctional F-actin and an average Pearson correlation coefficient \pm SEM is reported beneath the images. $n=50$ junctions over 5 fields of view. (c) Ankyrin $\mathrm{G}$ was immunoprecipitated and co-precipitation of E-cadherin was examined using immunoblotting. The graph beneath 
the blots indicates the average amount of E-cadherin recovered as a function of ankyrin $\mathrm{G}$ levels \pm SEM, $n=3$ biologically independent samples. (d-f) Inhibition of ankyrin $\mathrm{G}$ prevents GLUT1 enrichment in cell-cell junctions. MCF-10A cells expressing shRNAs targeting ankyrin $\mathrm{G}$ (shANKG) or a scramble sequence (scrANKG) were employed. (d) The immunoblots indicate the average amount of Ankyrin G normalized to loading control (vinculin) levels \pm SEM, $n=3$ biologically independent samples. (e and f). The cells were stained with antibodies against GLUT1 or $\beta$-catenin. Representative images are shown in e. The graph in $f$ represents the average corrected fluorescence intensity of junctional GLUT1 and an average Pearson correlation coefficient \pm SEM is reported beneath the images. $\mathrm{n}=50$ junctions over 5 fields of view. (g) Inhibition of ankyrin $\mathrm{G}$ prevents force stimulated glucose uptake. Glucose uptake was monitored as described in the legend of Figure 1a. The graphs represent the average glucose taken up into the cells \pm SEM, $\mathrm{n}=3$ (scrANKG), n=4 (shANKG), n=4 (Blebbistatin), n=3 (HECD-1). (h) Ankyrin $\mathrm{G}$ and GLUT1 co-immunoprecipitate with E-cadherin. Co-precipitation of GLUT1 and ankyrin G with E-cadherin was examined. The graphs beneath the immunoblots represent the average amount of GLUT1 or ankyrin G recovered as a function of the amount of E-cadherin \pm SEM. GLUT1/E-cad: n=3 (scrANKG/shANKG). ANKG/E-cad: $n=4$ (scrANKG), $n=3$ (shANKG). All immunofluorescence data are represented as a box and whisker plot with median, 10th, 25th, 75th, and 90th percentiles shown. Scale bars $=10 \mu \mathrm{M}$. For all experiments, significance was calculated using a two-tailed unpaired Student t-test. 

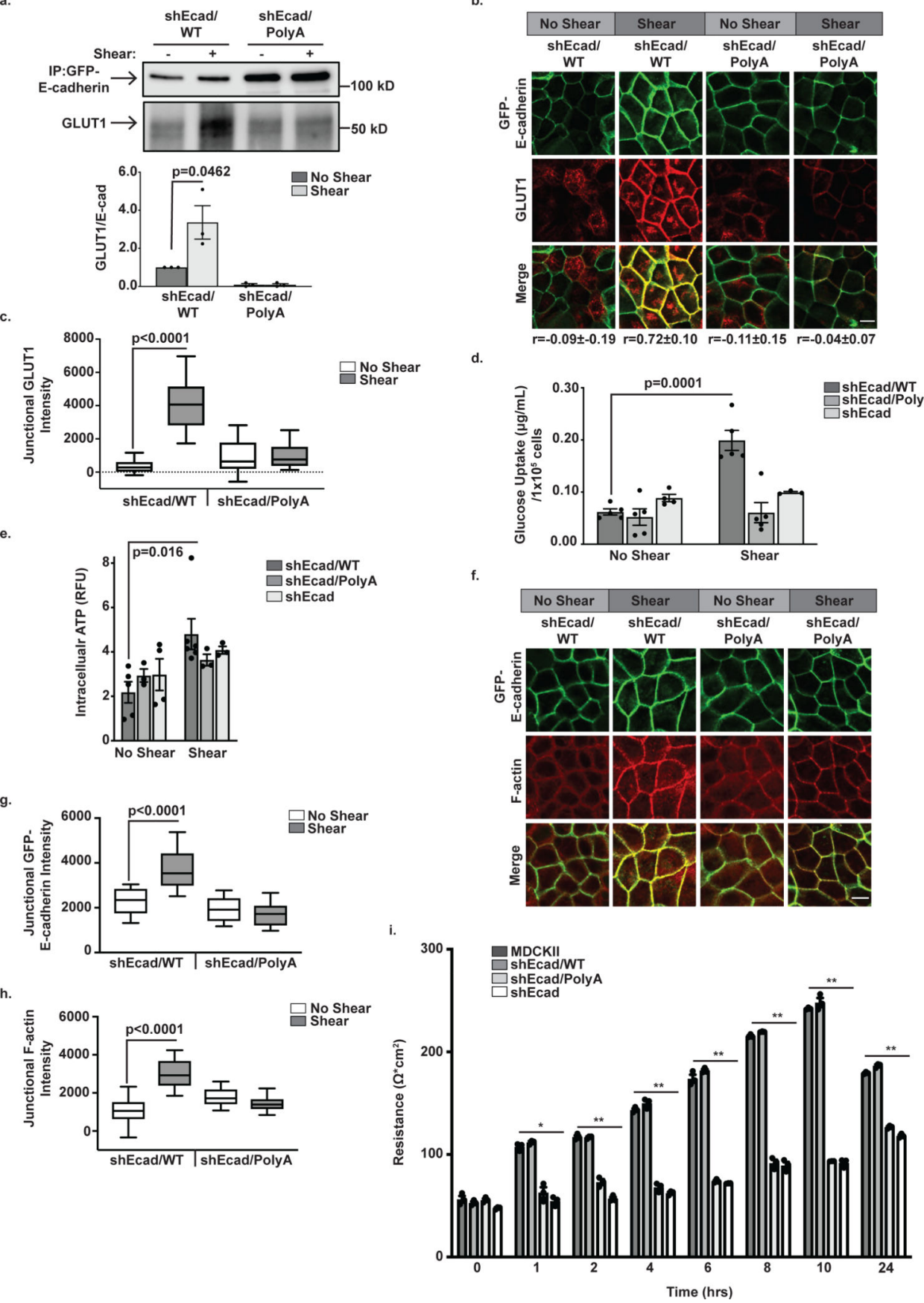

들

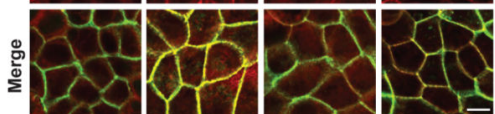

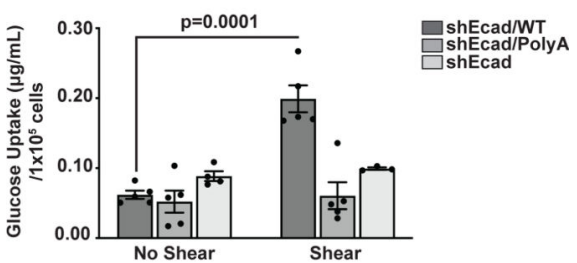

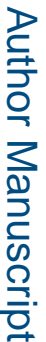

Figure 5: Ankyrin G binding to E-cadherin is required for force-stimulated glucose uptake, reinforcement of the actin cytoskeleton, and formation of an epithelial barrier.

MDCKII cells with depressed levels of E-cadherin were rescued with GFP tagged versions of wild-type E-cadherin (shEcad/WT) or a PolyA mutant E-cadherin unable to bind ankyrin G (shEcad/PolyA). (a-c) GLUT1 does not bind E-cadherin or localize to cell-cell junctions in cells expressing the PolyA mutant. (a) GFP-E-cadherin was immunoprecipitated and the co-precipitating levels of GLUT1 were examined. The graph beneath the immunoblot shows the average GLUT1 binding as a function of GFP-E-cadherin levels \pm SEM, $n=3$ biologically independent samples. (b-c) Cells were stained with antibodies against GLUT1,

Nat Cell Biol. Author manuscript; available in PMC 2021 September 09. 
and localization of GFP-E-cadherin and GLUT1 were examined. The graph in c represents the average corrected fluorescence intensity of junctional GLUT1. Scale bars $=10 \mu \mathrm{M} . n=50$ junctions over 5 fields of view (d-e) The PolyA mutant does not take up glucose or increase intercellular ATP levels in response to shear. (d) Glucose uptake was monitored and plotted as described in the Figure 1. $n=5(\operatorname{shEcad} / W T) n=5(\operatorname{shEcad} /$ PolyA), $n=3(\operatorname{shEcad})(e)$ Total relative ATP levels in cells were monitored. $n=6(\operatorname{shEcad} / W T), n=4$ (shEcad/PolyA), $\mathrm{n}=3$ (shEcad). Graphs represents average glucose uptake or intracellular ATP \pm SEM. (f-h) The PolyA mutant does not support actin cytoskeletal reinforcement. Localization of GFP-E-cadherin and F-actin were examined. Representative images are shown in $\mathrm{f}$. The graphs represent the average corrected fluorescence intensity of junctional GFP-E-cadherin (g) or F-actin (h). Scale bars $=10 \mu \mathrm{M} . \mathrm{n}=50$ junctions over 5 fields of view (i) Ankyrin $\mathrm{G}$ binding to E-cadherin is required for formation of a barrier. The cells were incubated in low calcium containing media. The formation of cell-cell junctions was stimulated by adding growth media to the cells. The trans-epithelial resistance across the epithelial monolayer was monitored at the indicated times (hours) after calcium re-addition. $\mathrm{n}=3$ biologically independent samples. All immunofluorescence data are represented as a box and whisker plot with median, 10th, 25th, 75th, and 90th percentiles shown. For all experiments, significance was calculated using a two-tailed unpaired Student t-test. * indicates $\mathrm{p} \leq 0.001$, ** indicates $\mathrm{p} \unlhd(0001$. 